\title{
11. MINERALOGY AND GEOCHEMISTRY OF CARBONATE DIAGENESIS OF THE PLIOCENE AND PLEISTOCENE HEMIPELAGIC MUD ON THE BLAKE OUTER RIDGE, SITE 533, LEG 76 1
}

\author{
Ryo Matsumoto, Geological Institute, Faculty of Science, University of Tokyo, Hongo, Tokyo, Japan
}

\begin{abstract}
Bulk X-ray mineralogy of 47 hemipelagic mud and clay samples from the Blake Outer Ridge has revealed that the sediments contain low magnesian calcite, calcian dolomite, ferroan dolomite, and magnesian siderite. Dolomite and siderite are authigenic and occur as rhombohedrons scattered through the sediments, whereas calcite is mostly biogenic. Pliocene dolomitic lenses are made up of interlocking polyhedral grains of ferroan dolomite. The contents of authigenic dolomite and siderite are 3 to $8 \%$ in carbonate sediments and 70 to $89 \%$ in dolomitic lenses. Dolomite occurs largely in the cores above $192 \mathrm{~m}$ sub-bottom depth, whereas siderite occurs in the cores below $87 \mathrm{~m}$. The distribution and occurrence of dolomite and siderite have determined the diagenetic zonation of carbonates as Zone I (dolomitic zone, top- 90 $\mathrm{m})$, Zone II (transition zone, 90-180 m), and Zone III (sideritic zone, $180 \mathrm{~m}$-bottom).

Measurements of major and minor elements in the untreated total sediment samples and the insoluble residues after digestion in acid-reducing solution have revealed that the soluble fraction concentrates carbonates and ferromanganese associations ( $\mathrm{Ca}, \mathrm{Mg}, \mathrm{Sr}, \mathrm{Fe}$, and $\mathrm{Mn}$ ). Typical "hydrogenous elements" ( $\mathrm{Co}, \mathrm{Cu}, \mathrm{Ni}$, and $\mathrm{V}$ ) are more concentrated in the insoluble residues rather than in the soluble fraction; the concentrations of these four elements are low and comparable to modern offshore mud, probably because the Site 533 sediments were deposited at a high rate of sedimentation. The contents of $\mathrm{Fe}_{2} \mathrm{O}_{3}$ and $\mathrm{MnO}$ are somewhat high for rapidly accumulated mud, particularly in the Pliocene sediments ( 8.09 and $0.26 \%$, respectively, on a carbonate-free basis). The high $\mathrm{Fe}$ and $\mathrm{Mn}$ contents are mainly due to the high contribution of the leacheable nonlithogenous fraction; leacheable $\mathrm{Fe}$ and $\mathrm{Mn}$ originate in the ferromanganese oxide accumulated on the seafloor. Only a small amount of ferric oxide was converted to iron sulfide in the surficial part of Zone I. Most ferromanganese oxide was reduced and precipitated as ferroan dolomite and magnesian siderite in Zones II and III under high alkalinity and high pH conditions in the organic-matter-rich sediments. $\mathrm{Fe}^{2+}$ and $\mathrm{Mn}^{2+}$ in the deeper sediments beneath Zone III possibly migrated upward and concentrated as siderite in Zone III, hence resulting in high contents of $\mathrm{Fe}$ and $\mathrm{Mn}$ in the Pliocene sediments.

Analysis of carbonate zonation on the Blake Outer Ridge has revealed that the zonation is subparallel to the bedding plane rather than to the present seafloor. The sediments at Site 103 on the flank region of the Ridge are lacking Zone I and most of Zone II, probably the result of erosion of the most of the Pleistocene and Pliocene sediments by the enhanced bottom currents during the Pleistocene.
\end{abstract}

\section{INTRODUCTION}

Organic-matter-rich hemipelagic mud and clay were taken from Site 533 on the crest of the Blake Outer Ridge, about $400 \mathrm{~km}$ off the eastern seaboard of the United States (Fig. 1). The sediments range in age from Holocene to middle Pliocene, with a distinct unconformity between the Pleistocene and Pliocene sediments. They are estimated to have accumulated at a high sedimentation rate of 7 to $21 \mathrm{~cm} / 1000 \mathrm{yr}$. Three holes were previously drilled on this Ridge during DSDP Leg 11 in 1972; authigenic carbonates in the form of disseminated rhombs, concretions, and nodules have been reported from the Neogene sediments (Zemmels et al., 1972). Moderately calcareous sediments at Site 533 also contain various amounts of authigenic dolomite and siderite besides biogenic calcite; dolomite tends to concentrate in the shallower cores and siderite in the deeper cores (as documented at Site 102 by Lancelot and Ewing, 1972).

There have been several studies done on the occurrence and origin of authigenic carbonates in the deepsea sediments. Matsumoto et al. (1978) analyzed ferroan dolomite and ankerite in the Albian to Aptian carbona-

\footnotetext{
${ }^{1}$ Sheridan, R. E., Gradstein, F. M., et al., Init, Repts. DSDP, 76: Washington (U.S.
} Govt. Printing Office) ceous shale at Sites 362 to 364 in the southeastern margin of the Atlantic, and attributed the high content of $\mathrm{FeCO}_{3}$ in dolomite to the iron that had been derived from the deeply weathered hinterland. Hein et al. (1979) studied the origin of the Neogene authigentic carbonates at Sites 184 to 192 in the deep Bering Sea and concluded that cations in carbonates originated from diagenetic alteration of volcanic ash. Matsumoto and Iijima (1980) reported authigenic high magnesian calcite and its derivative calcian dolomite and detrital zoned dolomite from Sites 438 and 439 off northeastern Japan. Zoned dolomite, with calcian dolomite core and ferroan dolomite or ankerite rim, have been frequently observed in marine sediments (e.g., Scholl, 1971; Matsumoto et al., 1978; Matsumoto and Iijima, 1980). Matsumota (1978) and Matsumoto and Iijima (1981) studied the origin of carbonates in coal measures of Japan and concluded that the variations of the species and chemical composition of authigenic carbonates reflect the fluctuation of the chemical composition of the pore waters caused by the migration and intermixing of the pore waters during burial.

It has been well documented that the deep-sea pelagic clay concentrates hydrogenous elements such as $\mathrm{Mn}, \mathrm{Fe}$, $\mathrm{Co}, \mathrm{Cu}, \mathrm{Ni}$, and $\mathrm{V}$ as ferromanganese oxide associations relative to the offshore mud (e.g., Matsumoto and Iiji$\mathrm{ma}$, in press). The behavior of the leacheable ferric ox- 

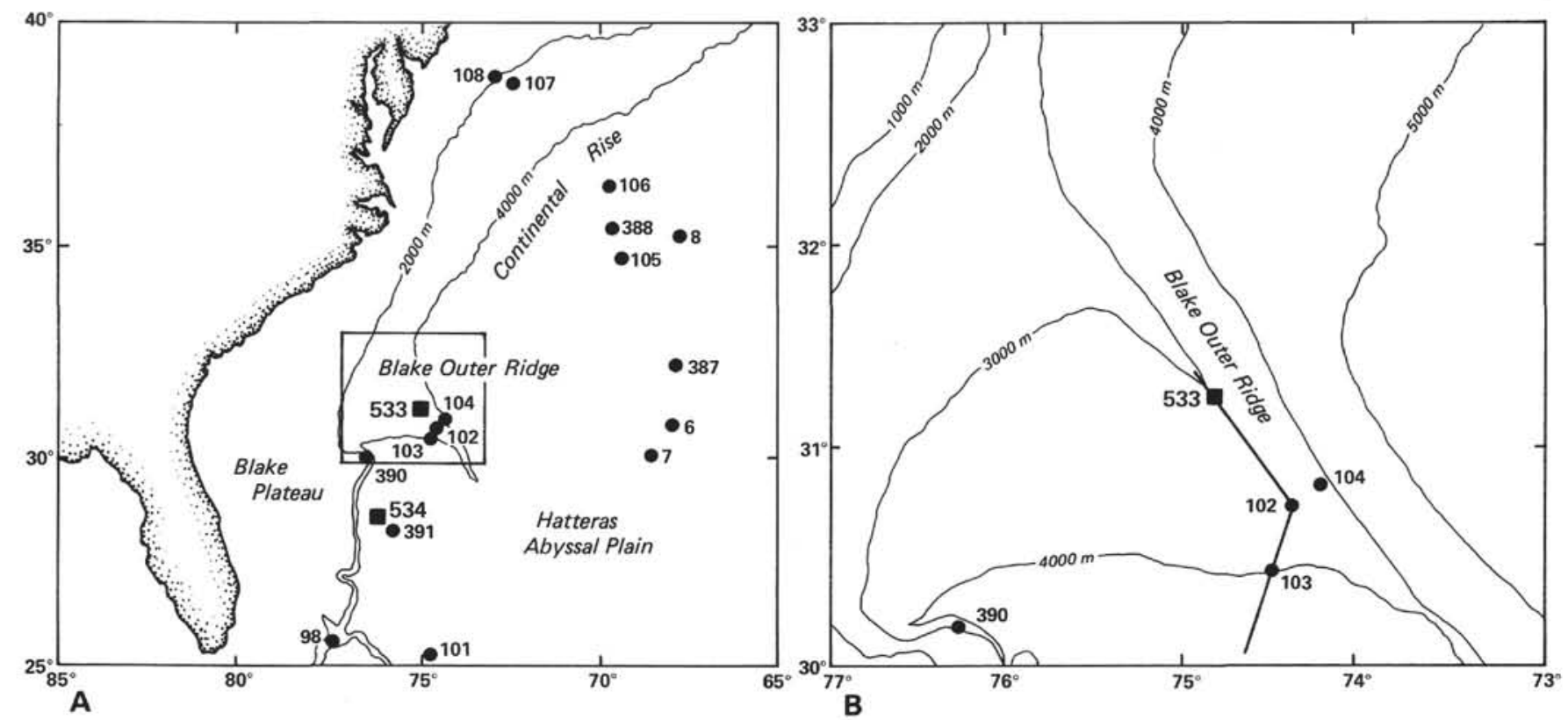

Figure 1. A and B. Location of DSDP Leg 76, Site 533 on the Blake Outer Ridge and other DSDP sites around the Ridge. (The solid line in Fig. 1B indicates the position of the schematic cross-section in Fig. 16.)

ide in marine sediments and the formation of iron minerals during burial have been generally discussed by Curtis (1977).

In this study, I discuss the origin of cations in the authigenic carbonates and the formative process of carbonate zonation in the rapidly accumulated, organicmatter-rich sediments in connection with the behavior of the initially incorporated ferromanganese oxide. Then I reexamine the construction of the Blake Outer Ridge based on the diagenetic zonation of carbonates; the Ridge was believed to have formed through accretion of hemipelagic mud and clay by geostrophic contour currents (e.g., Heezen et al., 1966; Ewing and Hollister, 1972).

\section{SAMPLES AND EXPERIMENTAL PROCEDURES}

Our investigation included 17 calcareous clay, mud, and silt samples from the Holocene to Pleistocene sections and 30 carbonate sediments from the Pliocene section. Except for two dolomitic lenses (Samples 533A-5-1, 10-15 cm and 533A-7-1, 16-18 cm), all samples are rather soft and poorly indurated and contain significant amounts of calcareous nannoplankton and foraminifers. The carbonate content varies widely from $11 \%$ (Sample $533-28-3,39-42 \mathrm{~cm}$ ) to $89 \%$ (Sample $533 \mathrm{~A}-7-1,16-18 \mathrm{~cm}$ ), but largely falls within the range of 15 and $36 \%$. The carbonate contents are, as a whole, higher in the Pleistocene sediments; the average carbonate content, except for two dolomitic lenses, is $28 \%$ (standard deviation [s.d.] $=12 \%$ ) for the Pleistocene and $22 \%$ (s.d. $=5 \%$ ) for the Pliocene sediments.

Semiquantitative X-ray powder diffraction analysis (XRD) was performed on all samples after desalination. The $d_{104}$ spacings of carbonate minerals in selected samples were accurately measured by XRD using silicon as an internal standard to estimate the deviation of the chemical composition of the carbonates from the end-member composition.

Concentrations of nine major elements- $\mathrm{Si}, \mathrm{Ti}, \mathrm{Al}, \mathrm{Fe}, \mathrm{Mg}, \mathrm{Ca}$, $\mathrm{Na}, \mathrm{K}, \mathrm{P}$-in total sediment samples were determined by wave dispersive X-ray fluorescence spectroscopy (XRF), Rigaku-Denki IKF 3064, with a chromium tube at $45 \mathrm{kV}$ and $30 \mathrm{~mA}$, using fused disc samples; ten minor elements- $\mathrm{Ba}, \mathrm{Co}, \mathrm{Cu}, \mathrm{Mn}, \mathrm{Ni}, \mathrm{Rb}, \mathrm{Sr}, \mathrm{V}, \mathrm{Zn}, \mathrm{Zr}$ - were also analyzed by the XRF with a tungsten tube at $70 \mathrm{kV}$ and $30 \mathrm{~mA}$, using pressed powder samples. Twenty-five samples were leached in an acid-reducing solution (hydroxylamine hydrochloride and acetic acid) following the method of Chester and Hughes (1967) to obtain the respective chemical composition of lithogenous insoluble residues and nonlithogenous soluble fractions. Concentrations of $\mathrm{Ti}, \mathrm{Ca}, \mathrm{Cu}$, $\mathrm{Mn}, \mathrm{Ni}$, and $\mathrm{Zn}$ in the insoluble residue were analyzed by energy dispersive XRF, Rigaku-Denki XES 0700 , with a rhodium tube at 40 to $60 \mathrm{kV}$ and 0.5 to $2 \mathrm{~mA} ; \mathrm{Fe}, \mathrm{K}, \mathrm{Ba}, \mathrm{Co}, \mathrm{Rb}$, and $\mathrm{Sr}$ were measured by wave dispersive XRF. The concentrations of insoluble $\mathrm{Si}$ and $\mathrm{Al}$ were calculated from the percentage of soluble fractions (carbonate content) and the concentrations in the total sediment samples.

Microtextures of soft carbonate sediments and the occurrence of the fine-grained carbonate minerals were observed with a scanning electron microscope (SEM), Hitachi S-400. The chemical composition of dolomite and calcite was determined with an X-ray microanalyzer (XMA), JEOL JXA-5, using carbonate minerals as standard samples.

\section{RESULTS}

\section{Bulk X-Ray Mineralogy and the Occurrence of Carbonates}

Carbonate minerals encountered in the Site 533 sediments are calcite, dolomite, and siderite. The abundances of these three carbonates were estimated from the peak heights on the X-ray diffractogram, as shown in Figure 2, in which the abundances of pyrite and clinoptilolite, and the percentage of the soluble fraction are also given.

\section{Calcite}

Calcite is ubiquitously distributed from the Holocene to the Pliocene sediments, predominating over dolomite and siderite in almost all cores (Fig. 2). The abundance of calcite is, as a whole, higher in the Pleistocene sediments, though widely fluctuating. The most calcareous samples (e.g., Sample 533-5-1, 100-103 cm) are estimated to contain about $50 \%$ calcite.

Calcite exhibits nearly constant $d_{104}$ spacing throughout the cores: The $d_{104}$ spacings are around $3.035 \AA$, the end-member value of pure calcite (Fig. 3). This value 


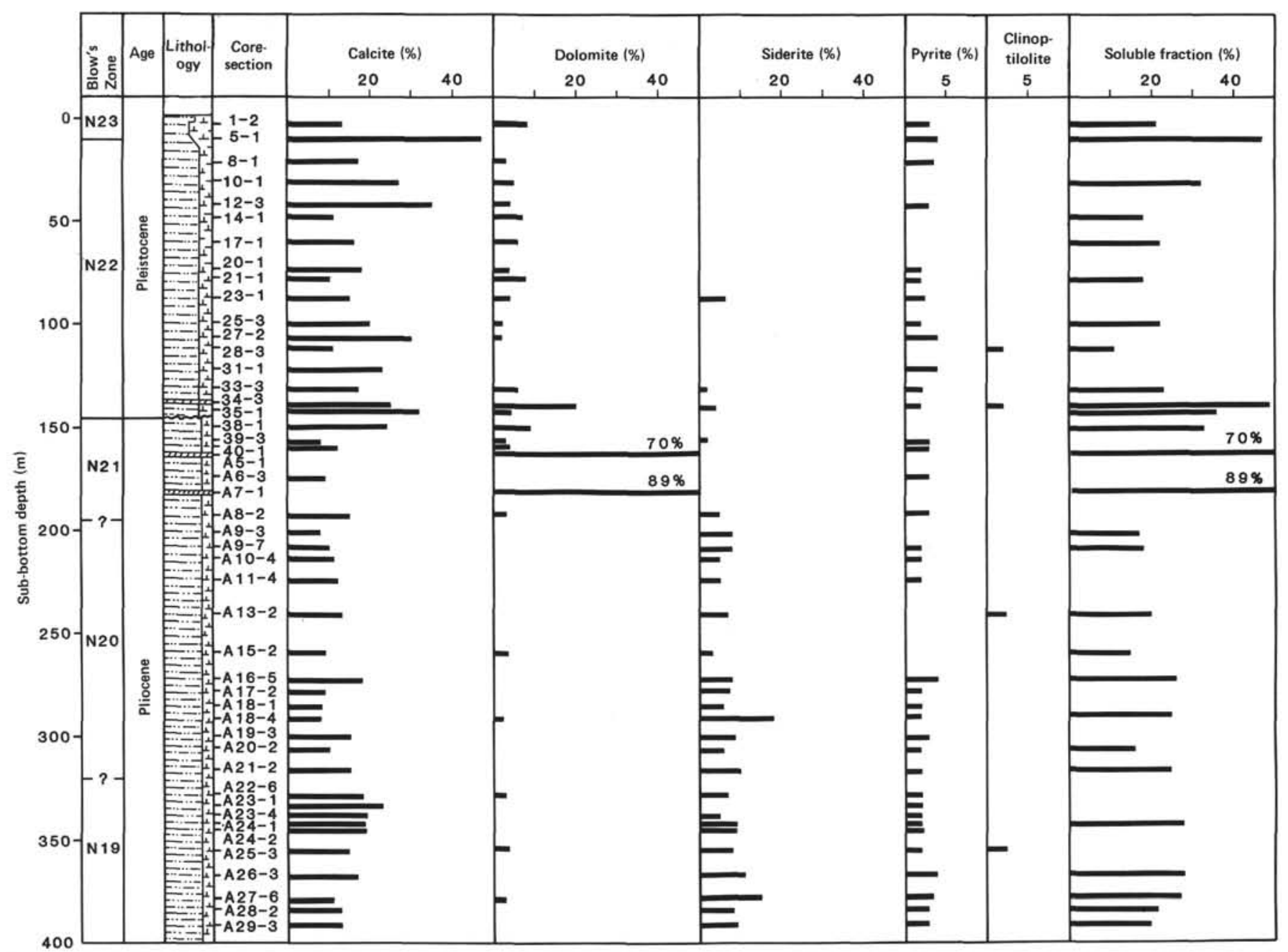

Figure 2. Distribution and abundances of calcite, dolomite, siderite, pyrite, and clinoptilolite in the Site 533 sediments. (The percentage of the soluble fraction given in the last column approximately indicates the carbonate contents.)

strongly suggests that calcite at Site 533 has nearly endmember composition. According to the previous data on the chemical composition of deep-sea calcite (e.g., Milliman and Müller, 1973; Hein et al., 1979; Matsumoto and Iijima, 1980), authigenic calcites in deep-sea sediments often exhibit high magnesium content, whereas that of biogenic calcite is usually low magnesian.

SEM observation has revealed that calcareous sediments contain abundant coccoliths and subordinate foraminiferal tests; authigenic calcite such as micrite or irregularly shaped grains filling interstices were very rare. These facts strongly suggest that most calcite at Site 533 is of biogenic rather than diagenetic origin. The preservation of calcareous fossils is usually moderate to good; beautiful coccolith shell structures are observed even in the Pliocene samples at deeper levels (Fig. 4A).

\section{Dolomite}

Dolomite is found in almost all cores above $192 \mathrm{~m}$ sub-bottom depth (Fig. 2). Even the shallowest sample $(533-1-2,50-53 \mathrm{~cm})$ at $2 \mathrm{~m}$ sub-bottom depth contains significant dolomite. In the deeper cores dolomite becomes less common, though it sporadically occurs in a few samples. The content of dolomite at Site 533 is estimated to be less than $10 \%$, except for the upper Pliocene dolomitic lenses, which contain 70 to $89 \%$ dolomite. Figure 5 shows the relation between the contents of calcite and dolomite plus siderite. As for Pleistocene dolomitic-calcitic samples, except for a highly dolomitic one (533-34-3, 101-103 cm), the contents of dolomite and calcite seem to correlate negatively. This negative correlation may suggest that dolomite originates from dolomitization of the preexisting biogenic calcite.

The $d_{104}$ spacing of dolomite ranges widely from $2.887 \AA$ to $2.906 \AA$ and seems to increases with depth (Fig. 3). Compared with the end-member value of dolomite $(2.886 \AA)$, the larger values of $d_{104}$ spacing suggests a variable degree of substitution of $\mathrm{Mg}$ by $\mathrm{Fe}$, or $\mathrm{Mg}$ by Ca.

Two different fabrics of dolomite were revealed by SEM observation. One is isolated rhombohedral to subhedral grains and the other is interlocking polyhedral grains. Rhombohedral dolomite, usually 0.005 to 0.035 $\mathrm{mm}$ in diameter, is ubiquitously disseminated in the dolomitic-calcitic samples. Smaller subhedral dolomite often associates with the rhombohedral dolomite. Rhom- 


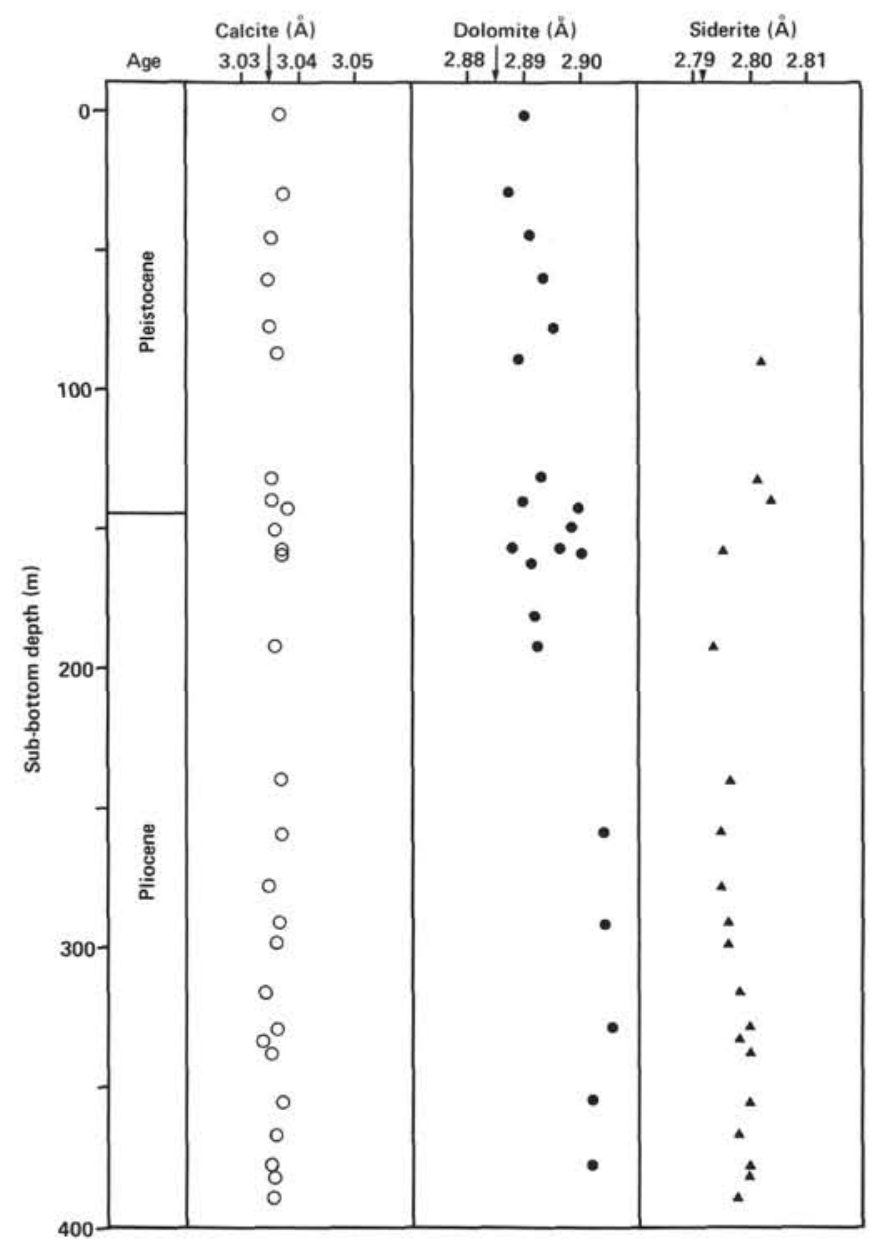

Figure 3. Fluctuations of $d_{104}$ spacing of calcite, dolomite, and siderite with depth at Site 533. (Arrows indicate the end-member value.)

bohedral and subhedral granular dolomite often exhibits characteristic rough appearance, which may suggest corrosion of the dolomite rhombs (Fig. 4B). The interlocking polyhedral dolomite is equant granular, about $0.003 \mathrm{~mm}$ in diameter, and exclusively contained in dolomitic lenses. The polyhedrons show smooth surfaces and sharp edges lacking corrosion, and sometimes exhibit crystal twins (Fig. 4C).

\section{Siderite}

Siderite appears first in Sample 533-23-1, 85-88 cm at $87 \mathrm{~m}$ sub-bottom depth, then sporadically occurs down to Sample $533 \mathrm{~A}-8-3,10-12 \mathrm{~cm}$ at $192 \mathrm{~m}$; beneath this level, siderite is observed continuously to the deepest sample (533A-29-3, 64-66 cm) at $390 \mathrm{~m}$ sub-bottom depth (Fig. 2). The content of siderite is estimated to be around $8 \%$ except for one sample (533A-18-4, 91-93 $\mathrm{cm})$, which contains about $18 \%$ siderite.

The $d_{104}$ spacing of siderite shows slight increase with depth from $2.795 \AA$ at $157 \mathrm{~m}$ to $2.800 \AA$ at $390 \mathrm{~m}$.

Siderite occurs as fine-grained rhombohedral grains, about $0.005 \mathrm{~mm}$ in diameter, scattered in moderately to sparsely calcareous sediments. Siderite rhombs in Section 533A-18-4 are surrounded by uneven surfaces, showing characteristic rhombic habits depicted in Figure 4D.

\section{Noncarbonates}

The common noncarbonate minerals encountered are quartz, plagioclase, K-feldspar, mica, and kaolinite. The abundance of these terrigenous minerals in the untreated total samples fluctuates widely, probably reflecting variable contents of carbonates. Quartz and feldspars, especially plagioclase, in the insoluble residues are more concentrated in the Holocene and Pleistocene sediments, compared with the Pliocene sediments. Chlorite and amphibole are restricted to the Pleistocene sediments, whereas montmorillonite and montmorillonite-illite mixed-layered clay are characteristic of the Pliocene sediments. A marked difference in bulk mineral composition between the Pleistocene and the Pliocene sediments is consistent with earlier findings at Site 102 by Zemmels et al. (1972); the higher contribution of terrigenous materials in the Pleistocene sediments probably reflects the drastic changes in the source of sediments and the process of sedimentation in the Pleistocene glacial period, as suggested by Ewing and Hollister (1972).

Trace amounts of pyrite were detected throughout the cores. It occurs as disseminated cubic grains, framboidal aggregates, or infilling of foraminiferal tests. Clinoptilolite only occurs in four samples beneath $112 \mathrm{~m}$ sub-bottom depth. Gypsum is found in the deepest sample alone.

\section{Major and Minor Element Geochemistry}

The results of XRF analyses of 47 untreated total samples and 25 lithogenous insoluble residues are given in Tables 1 and 2. Table 3 presents the concentrations of elements in the total samples $\left(C_{\mathrm{T}}\right)$, insoluble residues $\left(C_{\mathrm{IR}}\right)$, soluble fraction $\left(C_{\mathrm{SF}}\right)$, and the contribution of the soluble fraction $\left(R_{\mathrm{SF}}\right)$ on an average. The average compositions of modern pelagic clay, offshore mud, and calcareous nannofossil ooze are also shown for comparison. $C_{\mathrm{SF}}$ and $R_{\mathrm{SF}}$ were calculated as:

$$
\begin{gathered}
C_{\mathrm{SF}}=C_{\mathrm{T}}-C_{\mathrm{IF}}, \\
C_{\mathrm{IF}}=C_{\mathrm{IR}} \times(100-\mathrm{X}) / 100, \\
R_{\mathrm{SF}}=C_{\mathrm{SF}} / C_{\mathrm{T}},
\end{gathered}
$$

where $C_{\mathrm{IF}}$ is the concentration in the insoluble fraction of the total sediment samples, and $X$ is the percentage of soluble fraction estimated from the digestion treatment in the acid-reducing solution.

Major and minor elements in the sediments at Site 533 can be classified into two groups; those associated with soluble components and those associated with insoluble to poorly soluble components on the basis of the contribution of the soluble fraction $\left(R_{\mathrm{SF}}\right)$. Elements in the former group are $\mathrm{Fe}, \mathrm{Ca}, \mathrm{Mn}$, and $\mathrm{Sr}$. The components soluble in an acid-reducing solution are mainly carbonate minerals and ferromanganese oxide, and subordinately exchangeable ions adsorbed on clay minerals (Chester and Hughes, 1967). Therefore, soluble $\mathrm{CaO}$ and $\mathrm{Sr}$ are considered to have been derived chiefly from 


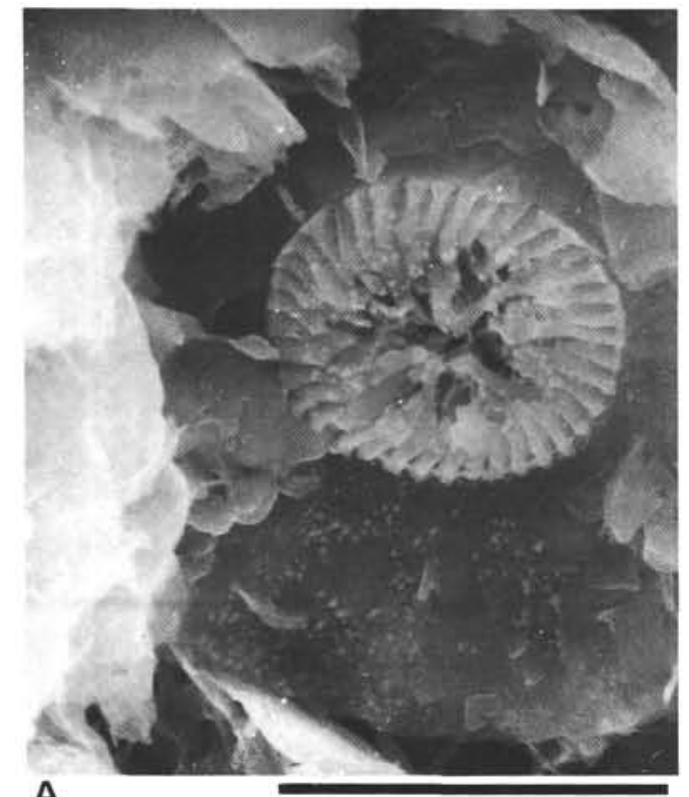

A

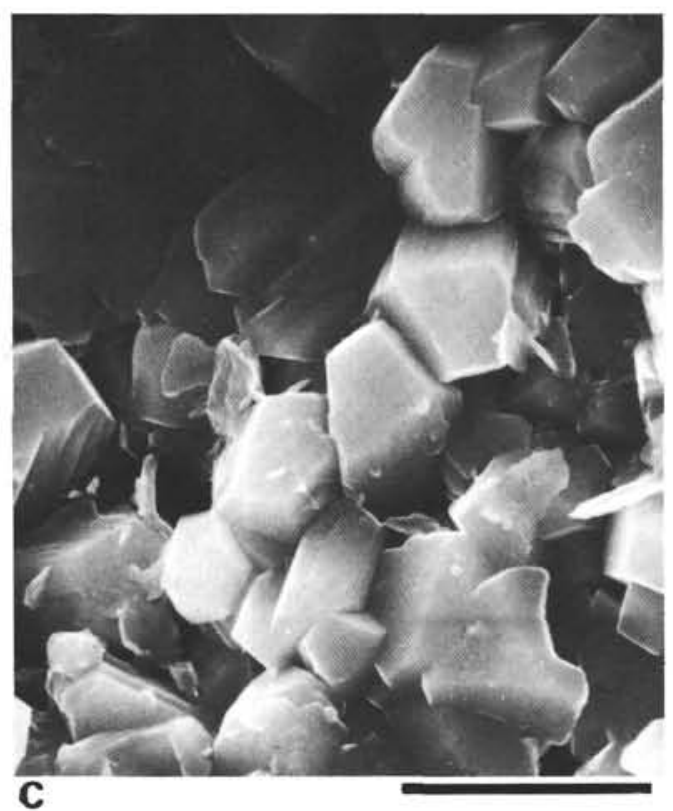

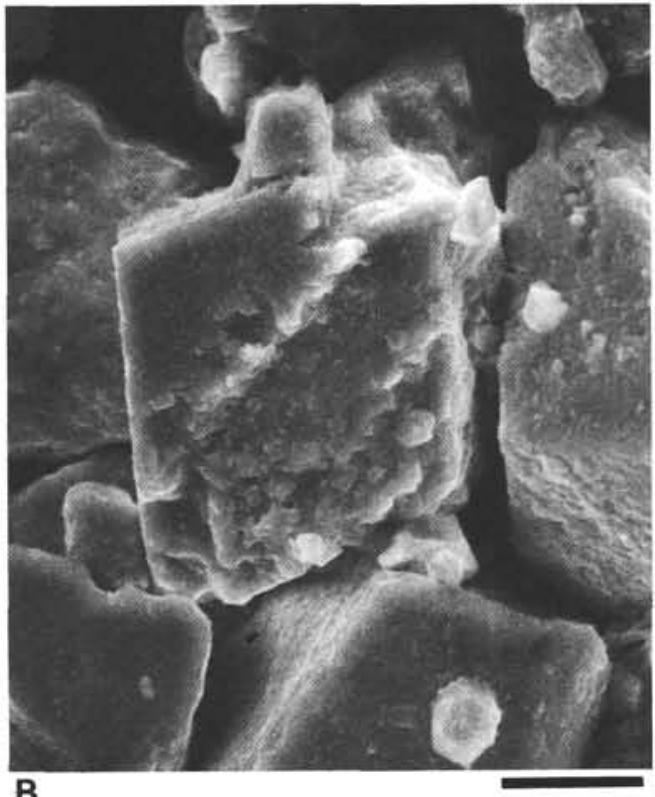

B

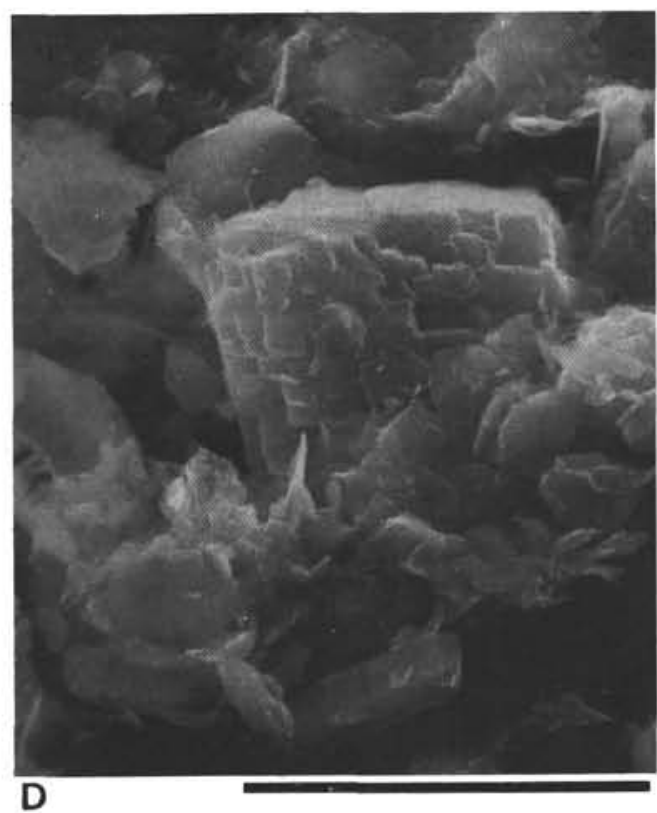

Figure 4. SEM photomicrographs of carbonate sediments at Site 533. (Scale bar $=0.005 \mathrm{~mm}$.) A. Well-preserved coccoliths in the sparsely calcareous sediments at $290.5 \mathrm{~m}$ sub-bottom depth in Zone III, Sample 533A-18-4, $91-93 \mathrm{~cm}$. B. Partly corroded rhombohedrons of calcian dolomite in Zone II, Sample 533-34-3, 101-103 cm. C. Interlocking polyhedrons of ferroan dolomite in Zone II, Sample 533A-7-1, 16-18 cm. D. Siderite crystal scattered in the sparsely calcareous sediments in Zone III, showing characteristic rhombic habit, Sample 533A-18-4, 91-93 cm.

calcite and dolomite, and $\mathrm{Fe}_{2} \mathrm{O}_{3}$ and $\mathrm{MnO}$, from siderite and ferromanganese oxide. Mg should probably be included in this group, considering the association with dolomite.

\section{$\mathrm{Ca}, \mathrm{Mg}$, and $\mathrm{Sr}$}

The contents of $\mathrm{MgO}$ and $\mathrm{Sr}$ are plotted against $\mathrm{CaO}$ content in total samples in Figures 6 and 7.

\section{$\mathrm{CaO}-\mathrm{MgO}$ Relation}

The $\mathrm{CaO}$ content in total samples ranges widely from $2.82 \%$ in sparsely calcareous mud (Section 533-28-3) to
$33.4 \%$ in dolomitic lens (Section 533A-5-1). By contrast, the $\mathrm{MgO}$ content mostly concentrates around $2.5 \%$, except for three highly dolomitic sections (533-34-3, 533A5-1, 533A-7-1). As shown in Figure 6, four calcitic sections (533-5-1, 533-28-3, 533-31-1, 533A-6-3) that contain only calcite as the carbonate phase show distinct negative correlation. The regression line is expressed as $\mathrm{MgO}=2.32-0.031 \mathrm{CaO}$. This line represents a trend of dilution of mud by low magnesian calcite. Substituting $56 \%$ for $\mathrm{CaO}$ in the equation, $\mathrm{MgO}$ becomes $0.58 \%$. This means that calcite $(\mathrm{CaO}=56 \%)$ includes only $0.58 \% \mathrm{MgO}\left(1.5 \mathrm{~mol}^{2} \mathrm{MgCO}_{3}\right)$. Substituting $0.98 \%$, 


\section{R. MATSUMOTO}

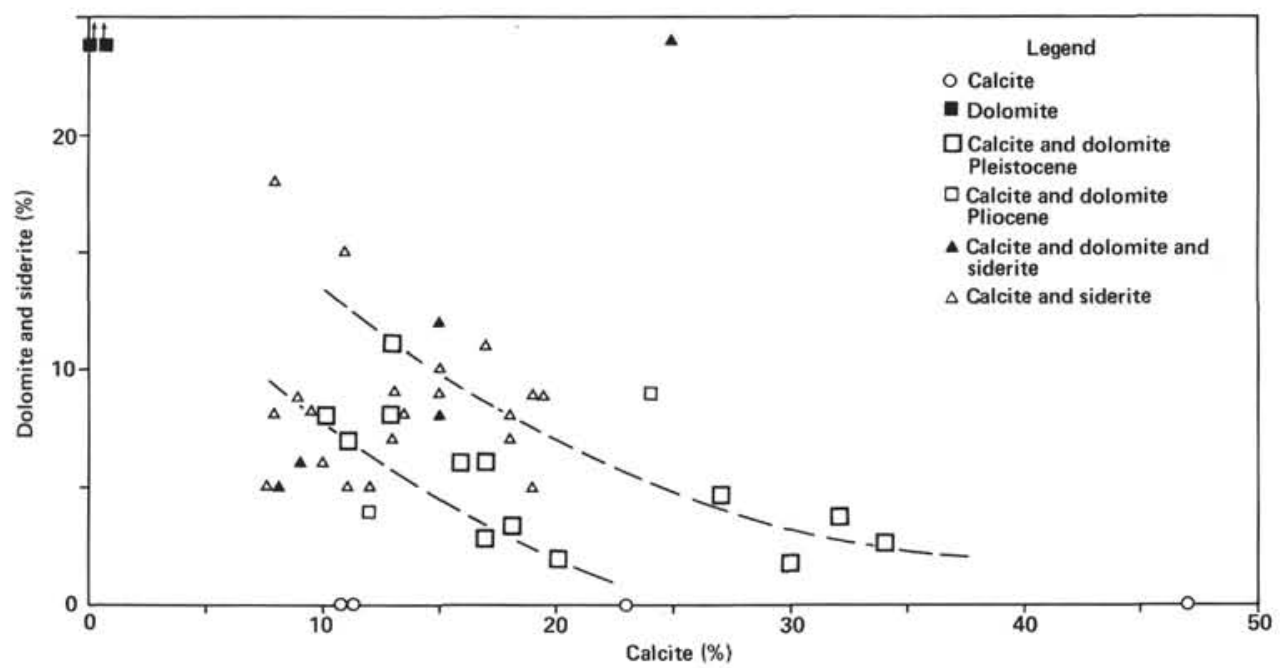

Figure 5. The aggregate content of dolomite and siderite plotted against the calcite content. (Dolomiticcalcitic samples in the Pleistocene sediments show negative correlation. Two dolomitic lenses are outside the diagram.)

Table 1. Major and minor element composition of the total sediment samples from Site 533 on the Blake Outer Ridge.

\begin{tabular}{|c|c|c|c|c|c|c|c|c|c|c|c|c|c|c|c|c|c|c|c|c|c|}
\hline \multirow{2}{*}{$\begin{array}{c}\text { Sample } \\
\text { (core-section, } \\
\text { interval in cm) }\end{array}$} & \multirow{2}{*}{$\begin{array}{l}\text { Sub-bottom } \\
\text { depth (m) }\end{array}$} & \multirow{2}{*}{$\begin{array}{l}\text { Carbonate } \\
\text { minerals }\end{array}$} & \multicolumn{8}{|c|}{ Major elements $(\%)$} & \multicolumn{11}{|c|}{ Minor elements (ppm) } \\
\hline & & & $\mathrm{SiO}_{2}$ & $\mathrm{TiO}_{2}$ & $\mathrm{Al}_{2} \mathrm{O}_{3}$ & $\mathrm{Fe}_{2} \mathrm{O}_{3}{ }^{*}$ & $\mathrm{MgO}$ & $\mathrm{CaO}$ & $\mathrm{Na}_{2} \mathrm{O}$ & $\mathrm{K}_{2} \mathrm{O}$ & $\mathrm{P}_{2} \mathrm{O}_{5}$ & $\mathrm{Ba}$ & Co & $\mathrm{Cu}$ & $\mathrm{Mn}$ & $\mathrm{Ni}$ & $\mathrm{Rb}$ & Sr & v & $\mathrm{Zn}$ & $\mathrm{Zr}$ \\
\hline \multicolumn{22}{|l|}{ Hole 533} \\
\hline $1-2,50-53$ & 2.0 & cal. + dol. & 54.59 & 0.72 & 13.53 & 5.43 & 3.22 & 8.61 & 2.13 & 3.18 & 0.13 & 335 & 14 & 35 & 656 & 34 & 100 & 229 & 85 & 71 & 190 \\
\hline $5-1,100-103$ & 9.4 & cal. & 35.91 & 0.43 & 8.61 & 3.29 & 1.63 & 24.69 & 1.41 & 1.84 & 0.13 & 183 & 8 & 92 & 636 & 26 & 51 & 820 & 45 & 231 & 180 \\
\hline $8-1,100-103$ & 21.4 & cal. + dol. & 44.92 & 0.59 & 12.82 & 7.30 & 2.58 & 13.25 & 1.41 & 2.85 & 0.15 & 321 & 16 & 26 & 1045 & 34 & 81 & 630 & 75 & 65 & 158 \\
\hline $10-1,100-103$ & 30.4 & cal. + dol. & 45.15 & 0.56 & 11.84 & 4.19 & 2.31 & 17.05 & 1.60 & 2.75 & 0.13 & 402 & 13 & 32 & 649 & 30 & 80 & 427 & 66 & 68 & 164 \\
\hline $12-3,48-51$ & 41.9 & cal. + dol. & 36.48 & 0.45 & 10.01 & 3.61 & 1.97 & 23.51 & 1.35 & 2.17 & 0.12 & 273 & 9 & 31 & 584 & 23 & 58 & 736 & 47 & 51 & 163 \\
\hline $14-1,37-40$ & 47.4 & cal. + dol. & 54.92 & 0.70 & 13.39 & 5.31 & 3.13 & 8.15 & 1.64 & 3.11 & 0.12 & 382 & 14 & 20 & 767 & 34 & 104 & 257 & 79 & 70 & 185 \\
\hline $17-1,80-81$ & 60.3 & cal. + dol. & 50.94 & 0.69 & 13.92 & 5.46 & 3.28 & 10.09 & 1.66 & 3.29 & 0.14 & 484 & 19 & 35 & 1049 & 35 & 97 & 269 & 82 & 69 & 164 \\
\hline $20-1,70-72$ & 73.7 & cal. + dol. & 47.43 & 0.52 & 11.94 & 5.14 & 3.10 & 13.15 & 2.21 & 2.58 & 0.13 & 477 & 13 & 28 & 794 & 43 & 74 & 485 & 67 & 75 & 127 \\
\hline $21-1,80-83$ & 78.3 & cal. + dol. & 53.46 & 0.59 & 13.19 & 5.10 & 3.13 & 9.51 & 2.60 & 2.81 & 0.13 & 586 & 17 & 29 & 742 & 35 & 81 & 353 & 76 & 68 & 152 \\
\hline $23-1,85-88$ & 87.3 & cal. + dol. + sid. & 53.89 & 0.74 & 14.81 & 6.01 & 3.31 & 9.61 & 1.49 & 3.29 & 0.13 & 505 & 15 & 10 & 951 & 32 & 104 & 252 & 84 & 71 & 164 \\
\hline $25-3,40-43$ & 98.9 & cal. + dol. & 45.39 & 0.54 & 12.61 & 5.27 & 2.87 & 14.71 & 1.89 & 2.60 & 0.13 & 347 & 17 & 31 & 940 & 42 & 77 & 567 & 59 & 76 & 146 \\
\hline $27-3,45-48$ & 106.5 & cal. + dol. & 34.79 & 0.42 & 9.34 & 3.80 & 1.88 & 23.89 & 1.31 & 2.01 & 0.15 & 314 & 9 & 47 & 623 & 23 & 58 & 843 & 44 & 57 & 190 \\
\hline $28-3,39-42$ & 112.4 & cal. & 55.19 & 0.93 & 17.89 & 7.56 & 2.21 & 2.82 & 1.34 & 3.28 & 0.09 & 387 & 20 & 32 & 837 & 42 & 129 & 150 & 113 & 95 & 190 \\
\hline $31-1,30-33$ & 122.8 & cal. & 45.50 & 0.55 & 11.47 & 4.88 & 1.70 & 16.11 & 1.28 & 2.27 & 0.11 & 294 & 13 & 59 & 454 & 37 & 72 & 579 & 59 & 122 & 176 \\
\hline $33-3,40-42$ & 131.9 & cal. + dol. + sid. & 48.45 & 0.60 & 14.33 & 5.91 & 3.33 & 9.29 & 2.25 & 3.13 & 0.13 & 443 & 21 & 35 & 886 & 50 & 97 & 339 & 74 & 84 & 126 \\
\hline $34-3,101-103$ & 140.0 & cal, + dol. + sid. & 35.30 & 0.39 & 8.61 & 4.22 & 5.65 & 22.16 & 1.02 & 2.09 & 0.11 & 192 & 8 & 0 & 704 & 21 & 46 & 164 & 37 & 40 & 97 \\
\hline $35-1,135-138$ & 141.9 & cal. + dol. & 42.09 & 0.54 & 10.94 & 4.35 & 2.10 & 19.22 & 1.20 & 2.32 & 0.11 & 298 & 7 & 31 & 379 & 25 & 71 & 474 & 64 & 64 & 172 \\
\hline $38-1,80-83$ & 150.3 & cal. + dol. + sid. & 45.88 & 0.59 & 11.93 & 5.03 & 3.37 & 14.23 & 1.27 & 2.52 & 0.12 & 372 & 11 & 44 & 466 & 35 & 85 & 389 & 68 & 73 & 167 \\
\hline $39-3,32-33$ & 157.3 & cal. + dol. & 54.15 & 0.71 & 14.22 & 5.80 & 2.48 & 5.62 & 1.16 & 2.65 & 0.13 & 241 & 14 & 42 & 375 & 44 & 100 & 236 & 100 & 105 & 170 \\
\hline $40-1,40-43$ & 158.9 & cal. + dol. & 51.45 & 0.67 & 14.55 & 6.05 & 2.58 & 8.29 & 0.94 & 2.56 & 0.15 & 327 & 14 & 28 & 450 & 44 & 100 & 335 & 88 & 92 & 149 \\
\hline \multicolumn{22}{|l|}{ Hole 533A } \\
\hline 5 & 161.6 & dol. & 3.09 & 0.11 & 1.80 & 1.65 & 18.45 & 33.43 & 0.29 & 0.45 & 0.0 & 254 & 2 & 0 & 1426 & 2 & 13 & 340 & 10 & 8 & 48 \\
\hline $6-3,40-42$ & 174.4 & cal. & 54.76 & 0.77 & 15.67 & 6.46 & 2.24 & 5.32 & 1.08 & 2.65 & 0.15 & 439 & 18 & 47 & 922 & 42 & 105 & 235 & 108 & 89 & 175 \\
\hline $7-1,16-18$ & 180.6 & dol. & 3.37 & 0.11 & 2.14 & 0.97 & 17.87 & 32.58 & 0.23 & 0.47 & 0.17 & 241 & 2 & 0 & 1538 & 3 & 16 & 337 & 11 & 10 & 52 \\
\hline $8-2,10-12$ & 192.1 & cal. + dol. + sid. & 50.58 & 0.66 & 13.58 & 5.93 & 2.00 & 9.29 & 1.07 & 2.54 & 0.14 & 354 & 15 & 36 & 632 & 44 & 96 & 375 & 85 & 85 & 162 \\
\hline $9-3,50-52$ & 203.0 & cal. + sid. & 51.78 & 0.71 & 16.53 & 6.17 & 2.84 & 5.88 & 1.26 & 2.48 & 0.15 & 429 & 23 & 49 & 3696 & 47 & 102 & 263 & 99 & 83 & 143 \\
\hline $9-7,108-110$ & 209.5 & cal. + sid. & 53.31 & 0.74 & 15.45 & 6.43 & 2.06 & 6.33 & 1.07 & 2.72 & 0.15 & 377 & 16 & 56 & 646 & 52 & 111 & 284 & 99 & 91 & 150 \\
\hline $10-4,70-72$ & 214.2 & cal. + sid. & 51.75 & 0.72 & 14.98 & 7.40 & 2.38 & 6.43 & 1.52 & 2.71 & 0.15 & 469 & 15 & 32 & 751 & 47 & 108 & 293 & 103 & 90 & 150 \\
\hline $11-4,70-72$ & 223.7 & cal. + sid. & 52.53 & 0.69 & 14.46 & 6.11 & 2.08 & 7.61 & 1.30 & 2.56 & 0.14 & 377 & 15 & 40 & 655 & 46 & 100 & 323 & 93 & 86 & 137 \\
\hline $13-2,98-100$ & 240.0 & cal. + sid. & 52.21 & 0.69 & 14.53 & 6.06 & 2.17 & 8.21 & 0.98 & 2.54 & 0.15 & 384 & 17 & 33 & 1105 & 48 & 101 & 340 & 89 & 95 & 149 \\
\hline $15-2,87-89$ & 258.9 & 1. + sid. & 54.29 & 0.80 & 17.53 & 6.18 & 2.25 & 5.62 & 1.07 & 2.53 & 0.14 & 401 & 19 & 51 & 1463 & 49 & 104 & 249 & 105 & 88 & 172 \\
\hline $16-5,60-62$ & 272.6 & 1. + sid. & 49.81 & 0.62 & 13.95 & 5.48 & 2.08 & 10.57 & 1.06 & 2.36 & 0.16 & 301 & 16 & 55 & 1159 & 45 & 88 & 407 & 80 & 82 & 143 \\
\hline $17-2,100-102$ & 278.0 & t sid & 53.76 & 0.73 & 16.14 & 6.24 & 2.35 & 6.18 & 1.16 & 2.5 & 0.15 & 262 & 18 & 48 & 1214 & 44 & 98 & 291 & 99 & 94 & 136 \\
\hline $18-1,60-62$ & 285.6 & cal. + sid. & 52.77 & 0.75 & 17.02 & 7.01 & 2.36 & 4.75 & 1.23 & 2.56 & 0.14 & 406 & 19 & 56 & 1816 & 49 & 99 & 239 & 107 & 89 & 142 \\
\hline $18-4,91-93$ & 290.5 & cal. + dol. + sid. & 47.96 & 0.67 & 14.74 & 10.60 & 3.78 & 5.07 & 1.26 & 2.30 & 0.18 & 389 & 16 & 36 & 4846 & 32 & 79 & 203 & 110 & 72 & 114 \\
\hline $19-3,100-102$ & 298.5 & cal. + sid. & 51.57 & 0.72 & 16.12 & 6.53 & 2.31 & 8.11 & 1.05 & 2.58 & 0.16 & 348 & 17 & 37 & 1728 & 48 & 104 & 334 & 95 & 101 & 143 \\
\hline $20-2,50-52$ & 306.0 & cal. + sid. & 51.99 & 0.72 & 17.29 & 6.25 & 2.46 & 5.63 & 1.21 & 2.49 & 0.13 & 387 & 22 & 55 & 2221 & 50 & 101 & 276 & 113 & 91 & 137 \\
\hline $21-2,102-104$ & 316.0 & $\mathrm{cal} .+\mathrm{si}$ & 49.32 & 0.65 & 13.89 & 5.63 & 2.05 & 8.91 & 1.22 & 2.37 & 0.15 & 345 & 14 & 61 & 1510 & 50 & 92 & 367 & 96 & 87 & 148 \\
\hline $22-6,102-104$ & 328.5 & cal. + dol. + sid. & 47.97 & 0.63 & 13.81 & 5.77 & 2.25 & 11.04 & 0.87 & 2.34 & 0.16 & 329 & 16 & 38 & 1766 & 42 & 87 & 427 & 89 & 82 & 146 \\
\hline $23-1,10-12$ & 330.5 & cal. + sid. & 47.25 & 0.61 & 13.60 & 5.11 & 1.93 & 13.36 & 1.07 & 2.32 & 0.18 & 283 & 13 & 32 & 1389 & 42 & 87 & 475 & 82 & 80 & 144 \\
\hline $23-4,58-60$ & 333.1 & cal. + sid. & 49.12 & 0.66 & 14.30 & 5.50 & 2.17 & 10.54 & 1.02 & 2.44 & 0.16 & 342 & 13 & 32 & 1279 & 42 & 94 & 413 & 92 & 83 & 149 \\
\hline $24-1,100-102$ & 343.0 & cal. + sid. & 47.25 & 0.64 & 14.06 & 5.66 & 2.21 & 11.23 & 1.07 & 2.36 & 0.18 & 316 & 14 & 43 & 1533 & 37 & 81 & 426 & 92 & 85 & 148 \\
\hline $24-2,56-58$ & 344.0 & cal. + sid & 48.22 & 0.64 & 14.05 & 5.77 & 2.21 & 11.01 & 0.96 & 2.48 & 0.19 & 301 & 11 & 53 & 1474 & 39 & 90 & 407 & 85 & 81 & 148 \\
\hline $25-3,40-42$ & 354.9 & cal. + dol. + sid. & 49.69 & 0.68 & 15.10 & 6.48 & 2.26 & 8.30 & 1.15 & 2.41 & 0.13 & 327 & 20 & 44 & 1966 & 47 & 83 & 348 & 103 & 86 & 142 \\
\hline $26-3,111-113$ & 366.5 & $\mathrm{cal} .+\mathrm{si}$ & 48.30 & 0.68 & 14.75 & 5.87 & 2.32 & 9.45 & 0.94 & 2.46 & 0.21 & 339 & 18 & 36 & 1538 & 41 & 89 & 374 & 96 & 94 & 150 \\
\hline $27-6,29-31$ & 378.2 & cal. + dol. + sid. & 52.92 & 0.73 & 16.02 & 6.45 & 2.39 & 6.01 & 1.17 & 2.60 & 0.16 & 381 & 18 & 47 & 1743 & 46 & 101 & 315 & 112 & 107 & 153 \\
\hline $64-66$ & 382.1 & & 52.71 & 0.74 & & 6.36 & 2.60 & 8.06 & 0.8 & 2.54 & 0.18 & 373 & 15 & 42 & 1823 & 46 & 101 & 315 & 112 & 107 & 153 \\
\hline $29-3,64-66$ & 390.0 & cal. + sid. & 49.94 & 0.72 & 15.10 & 6.68 & 2.60 & 7.73 & 1.03 & 2.71 & 0.27 & 400 & 17 & 53 & 2606 & 39 & 102 & 319 & 107 & 87 & 145 \\
\hline
\end{tabular}

Note: $\mathrm{Fe}_{2} \mathrm{O}_{3}{ }^{*}=$ total iron as $\mathrm{Fe}_{2} \mathrm{O}_{3} ;$ cal. = calcite; dol. = dolomite; sid. = siderite. 
Table 2. Major and minor element composition of the insoluble residues of the Site 533 sediments.

\begin{tabular}{|c|c|c|c|c|c|c|c|c|c|c|c|c|c|c|}
\hline \multirow{2}{*}{$\begin{array}{c}\text { Sections } \\
\text { (core-section) }\end{array}$} & \multicolumn{6}{|c|}{ Major elements $(\%)$} & \multicolumn{8}{|c|}{ Minor elements (ppm) } \\
\hline & $\mathrm{SiO}_{2}$ & $\mathrm{TiO}_{2}$ & $\mathrm{Al}_{2} \mathrm{O}_{3}$ & $\mathrm{Fe}_{2} \mathrm{O}_{3}{ }^{*}$ & $\mathrm{CaO}$ & $\mathrm{K}_{2} \mathrm{O}$ & $\mathrm{Ba}$ & $\mathrm{Co}$ & $\mathrm{Cu}$ & $\mathrm{Mn}$ & $\mathrm{Ni}$ & $\mathrm{Rb}$ & $\mathrm{Sr}$ & $\mathrm{Zn}$ \\
\hline \multicolumn{15}{|l|}{ Hole 533} \\
\hline $1-2$ & 69.10 & 1.07 & 17.13 & 6.15 & 0.69 & 3.52 & 418 & 25 & 26 & 477 & 40 & 171 & 106 & 100 \\
\hline $5-1$ & 67.75 & 0.94 & 16.24 & 7.56 & 1.09 & 2.36 & 427 & 33 & 75 & 479 & 47 & 100 & 203 & 110 \\
\hline $10-1$ & 66.40 & 0.98 & 17.41 & 5.70 & 0.96 & 3.03 & 444 & 40 & 16 & 482 & 61 & 112 & 107 & 99 \\
\hline $14-1$ & 66.97 & 0.91 & 16.33 & 5.63 & 0.83 & 3.66 & 356 & 15 & 10 & 367 & 43 & 121 & 109 & 81 \\
\hline $17-1$ & 65.31 & 0.81 & 17.85 & 6.68 & 1.37 & 3.60 & 527 & 24 & 28 & 550 & 43 & 120 & 178 & 88 \\
\hline $21-1$ & 65.19 & 0.92 & 16.07 & 7.00 & 2.36 & 3.64 & 433 & 21 & 47 & 549 & 57 & 79 & 323 & 95 \\
\hline $25-3$ & 63.04 & 0.98 & 17.54 & 5.94 & 2.04 & 3.64 & 480 & 22 & 51 & 691 & 84 & 111 & 192 & 103 \\
\hline $28-3$ & 62.00 & 1.14 & 20.10 & 5.92 & 0.56 & 3.61 & 289 & 22 & 33 & 372 & 50 & 158 & 83 & 94 \\
\hline $33-3$ & 62.92 & 0.84 & 18.61 & 7.76 & 1.54 & 3.22 & 381 & 28 & 51 & 600 & 68 & 106 & 209 & 114 \\
\hline $34-3$ & 69.22 & 0.85 & 16.88 & 5.02 & 2.07 & 3.49 & 472 & 21 & 22 & 467 & 48 & 98 & 156 & 86 \\
\hline $35-1$ & 65.76 & 0.91 & 17.09 & 8.34 & 1.23 & 2.83 & 390 & - & 34 & 411 & 43 & - & - & 98 \\
\hline $38-1$ & 68.48 & 0.77 & 17.80 & 6.99 & 1.45 & 2.53 & 410 & - & 37 & 395 & 43 & - & - & 90 \\
\hline \multicolumn{15}{|l|}{ Hole $533 \mathrm{~A}$} \\
\hline $9-3$ & 62.39 & 0.95 & 19.91 & 7.25 & 0.81 & 2.65 & 334 & 17 & 44 & 432 & 51 & 160 & 53 & 82 \\
\hline 9.7 & 65.00 & 1.06 & 18.84 & 6.36 & 0.66 & 3.33 & 272 & 23 & 76 & 352 & 64 & 144 & 82 & 101 \\
\hline $13-2$ & 65.26 & 0.78 & 18.16 & 5.70 & 0.45 & 3.59 & 235 & 15 & 66 & 353 & 53 & 146 & 76 & 87 \\
\hline $15-2$ & 63.87 & 1.19 & 20.62 & 6.35 & 0.69 & 3.38 & 405 & 15 & 59 & 305 & 55 & 138 & 90 & 88 \\
\hline $16-5$ & 67.31 & 0.96 & 18.85 & 5.38 & 0.91 & 3.30 & 287 & 12 & 71 & 340 & 54 & 160 & 86 & 96 \\
\hline $18-4$ & 64.81 & 1.08 & 19.92 & 5.83 & 0.77 & 2.84 & 375 & 23 & 165 & 386 & 46 & 119 & 90 & 95 \\
\hline $20-2$ & 61.90 & 0.92 & 20.59 & 5.47 & 0.51 & 2.60 & 330 & 26 & 62 & 310 & so & 158 & 64 & 108 \\
\hline $21-2$ & 65.77 & 0.87 & 18.52 & 5.64 & 0.81 & 2.36 & 337 & 2 & 176 & 387 & 43 & 140 & 98 & 117 \\
\hline $23-1$ & 65.63 & 0.99 & 18.89 & 6.48 & 0.86 & 3.07 & 332 & 20 & 101 & 400 & 47 & 177 & 78 & 103 \\
\hline $24-1$ & 65.63 & 1.07 & 19.53 & 6.49 & 0.57 & 2.90 & 350 & 17 & 70 & 414 & 56 & 162 & 115 & 94 \\
\hline $26-3$ & 65.27 & 1.14 & 19.93 & 5.97 & 0.56 & 3.01 & 310 & 14 & 46 & 403 & 57 & 170 & 60 & 93 \\
\hline $27-6$ & 66.99 & 0.94 & 20.28 & 7.35 & 0.47 & 2.31 & 412 & 10 & 50 & 507 & 50 & 169 & 88 & 93 \\
\hline $29-3$ & 62.43 & 1.06 & 18.88 & 5.42 & 0.88 & 3.93 & 270 & 9 & 43 & 357 & 48 & 166 & 73 & 92 \\
\hline
\end{tabular}

Table 3. The average partitioning of major and minor elements in Site 533 Pleistocene and Pliocene sediments and the average composition of recent deep-sea sediments (number of samples indicated within parentheses).

\begin{tabular}{|c|c|c|c|c|c|c|c|c|c|c|c|}
\hline & \multicolumn{2}{|c|}{$\begin{array}{c}\text { Total samples } \\
\left(C_{\mathrm{T}}\right)\end{array}$} & \multicolumn{2}{|c|}{$\begin{array}{c}\text { Insoluble residue } \\
\left(C_{I R}\right)\end{array}$} & \multicolumn{2}{|c|}{$\begin{array}{c}\text { Soluble fraction } \\
\left(C_{\mathrm{SF}}\right)\end{array}$} & \multicolumn{2}{|c|}{$\begin{array}{l}\text { Contribution of } \\
\text { soluble fraction } \\
\end{array}$} & \multicolumn{3}{|c|}{$\begin{array}{l}\text { Recent deep-sea } \\
\text { sediments }{ }^{\mathrm{a}}\end{array}$} \\
\hline & $\begin{array}{c}\text { Pleist. } \\
\text { (17) }\end{array}$ & $\begin{array}{l}\text { Plioc. } \\
\text { (28) }\end{array}$ & $\begin{array}{l}\text { Pleist. } \\
\text { (11) }\end{array}$ & $\begin{array}{c}\text { Plioc. } \\
\text { (14) }\end{array}$ & $\begin{array}{l}\text { Pleist. } \\
\text { (11) }\end{array}$ & $\begin{array}{c}\text { Plioc. } \\
\text { (14) }\end{array}$ & $\begin{array}{l}\text { Pleist. } \\
\text { (11) }\end{array}$ & $\begin{array}{l}\text { Plioc. } \\
\text { (14) }\end{array}$ & $\begin{array}{l}\text { Offshore } \\
\text { mud } \\
\text { (3) }\end{array}$ & $\begin{array}{l}\text { Pelagic } \\
\text { clay } \\
\text { (6) }\end{array}$ & $\begin{array}{c}\text { Nannofossil } \\
\text { ooze } \\
\text { (3) }\end{array}$ \\
\hline $\mathrm{SiO}_{2}$ & 46.14 & 50.83 & $(65.79)$ & $(65.05)$ & (0) & (0) & (0) & (0) & 67.43 & 58.89 & 19.30 \\
\hline $\mathrm{TiO}_{2}$ & 0.59 & 0.69 & 0.94 & 0.98 & 0 & 0 & 0 & 0 & 0.59 & 0.74 & 0.23 \\
\hline $\mathrm{Al}_{2} \mathrm{O}_{3}$ & 12.31 & 14.97 & (17.39) & $(19.56)$ & (0) & (0) & (0) & (0) & 13.05 & 15.57 & 5.79 \\
\hline $\mathrm{Fe}_{2} \mathrm{O}_{3} \cdot$ & 5.11 & 6.25 & 6.56 & 6.19 & 0.55 & 1.37 & 6 & 22 & 5.39 & 7.14 & 2.13 \\
\hline $\mathrm{MgO}$ & 2.79 & 2.39 & - & - & - & - & - & - & 2.25 & 2.93 & 1.24 \\
\hline $\mathrm{CaO}$ & 14.46 & 8.17 & 1.34 & 0.74 & 12.33 & 7.23 & 91 & 93 & 2.04 & 1.80 & 37.41 \\
\hline $\mathrm{Na}_{2} \mathrm{O}$ & 1.63 & 1.11 & - & - & - & - & - & - & 2.09 & 2.29 & 0.94 \\
\hline $\mathrm{K}_{2} \mathrm{O}$ & 2.68 & 2.51 & 3.33 & 2.99 & 0.35 & 0.26 & 13 & 7 & 1.86 & 2.97 & 1.25 \\
\hline $\mathrm{P}_{2} \mathrm{O}_{5}$ & 0.13 & 0.16 & - & - & - & - & - & - & 0.04 & 0.22 & 0.02 \\
\hline $\mathrm{Ba}$ & 366 & 357 & 420 & 333 & 75 & 105 & 13 & 30 & 529 & 692 & 1181 \\
\hline Co & 14 & 16 & 25 & 16 & 0.3 & 5 & 0 & 24 & 11 & 64 & 14 \\
\hline $\mathrm{Cu}$ & 34 & 44 & 36 & 76 & 13 & 5 & 24 & 12 & 92 & 237 & 60 \\
\hline $\mathrm{Mn}$ & 747 & 1528 & 495 & 381 & 392 & 1558 & 51 & 78 & 630 & 6504 & 2038 \\
\hline $\mathrm{Ni}$ & 33 & 44 & 53 & 51 & 0.5 & 5 & 0 & 10 & 35 & 115 & 14 \\
\hline $\mathrm{Rb}$ & 82 & 96 & 118 & 154 & 4 & 0 & 3 & 0 & 70 & 99 & 30 \\
\hline $\mathrm{Sr}$ & 446 & 330 & 167 & 81 & 237 & 265 & 60 & 80 & 157 & 152 & 770 \\
\hline V & 68 & 88 & - & - & - & - & - & - & 87 & 120 & 44 \\
\hline $\mathrm{Zn}$ & 81 & 88 & 97 & 96 & 2 & 11 & 0 & 13 & 135 & 111 & 5 \\
\hline $\mathrm{Zr}$ & 161 & 148 & - & - & - & - & - & - & 123 & 141 & 120 \\
\hline
\end{tabular}

Note: Values in parentheses were calculated from $C_{\mathrm{T}}$ and the percentage of the soluble fraction; -indicates no data available.

a Matsumoto and lijima (in press).

the average of $\mathrm{CaO}$ contents of the insoluble residues, $\mathrm{MgO}$ becomes $2.3 \%$. This value is regarded as an average $\mathrm{MgO}$ content of the insoluble residue.

\section{CaO-Sr Relation}

The $\mathrm{Sr}$ content in total samples averages $427 \mathrm{ppm}$ for the Pleistocene sediments and $331 \mathrm{ppm}$ for the Pliocene sediments. The higher value in the former corresponds to the high content of calcite. Both values fall between the range of noncalcareous deep-sea clay and calcareous nannofossil ooze (Table 3). Four calcitic sections (5335-1, 533-28-3, 533-31-1, 533A-6-3) exhibit marked positive relation, as shown in Figure 7 . The regression line is
$\mathrm{Sr}=0.00701+0.00307 \mathrm{CaO}$, which indicates that calcite contains about $1800 \mathrm{ppm} \mathrm{Sr}$. Estimation from the contents of soluble $\mathrm{CaO}$ and $\mathrm{Sr}$ in the calcitic samples gives about $1700 \mathrm{ppm} \mathrm{Sr}$ in calcite. According to Thompson and Bowen (1969), Atlantic deep-sea coccoliths are estimated to contain about $1500 \mathrm{ppm} \mathrm{Sr}$, which is fairly close to the present data. $\mathrm{Mg}$ and $\mathrm{Sr}$ contents in calcite support the SEM observation that most calcite at Site 533 is biogenic. Pliocene samples containing both calcite and siderite plot near the regression line, indicating that the $\mathrm{Sr} / \mathrm{CaO}$ ratio of siderite is similar to that of biogenic calcite. Dolomitic lenses exhibit lower $\mathrm{Sr} / \mathrm{CaO}$ ratios, about one-third that of calcite, which suggests that the dolomite contains only $340 \mathrm{ppm} \mathrm{Sr}$. 


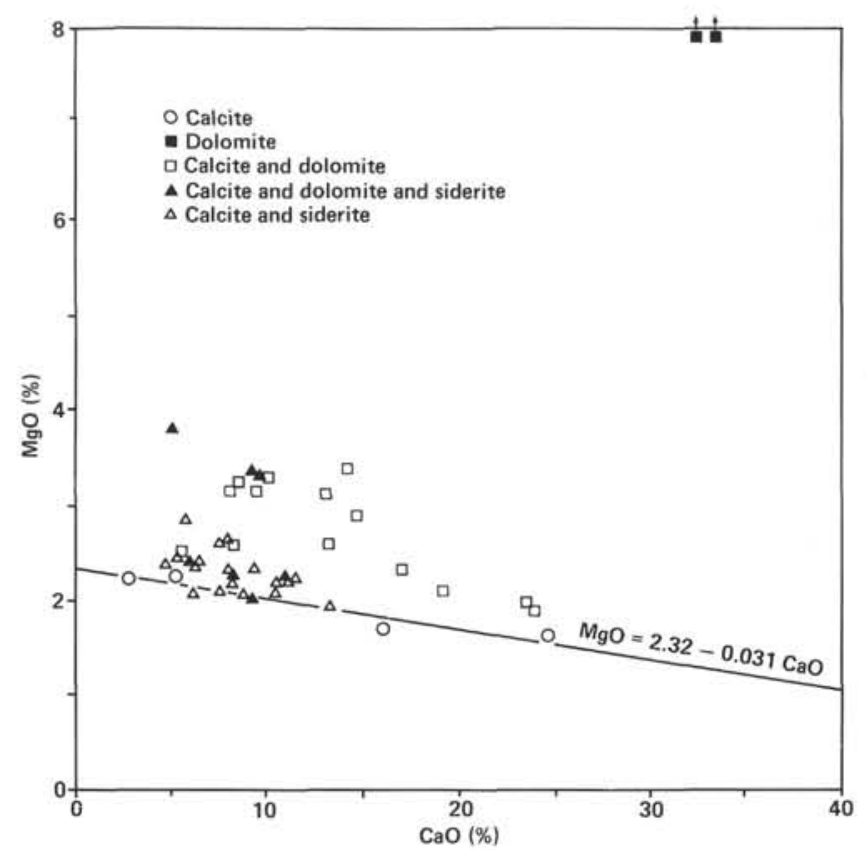

Figure 6. Correlation between the contents of $\mathrm{MgO}$ and $\mathrm{CaO}$ in the untreated total sediment samples. (Four calcitic samples show negative correlation. Two dolomitic lenses are outside the diagram.)

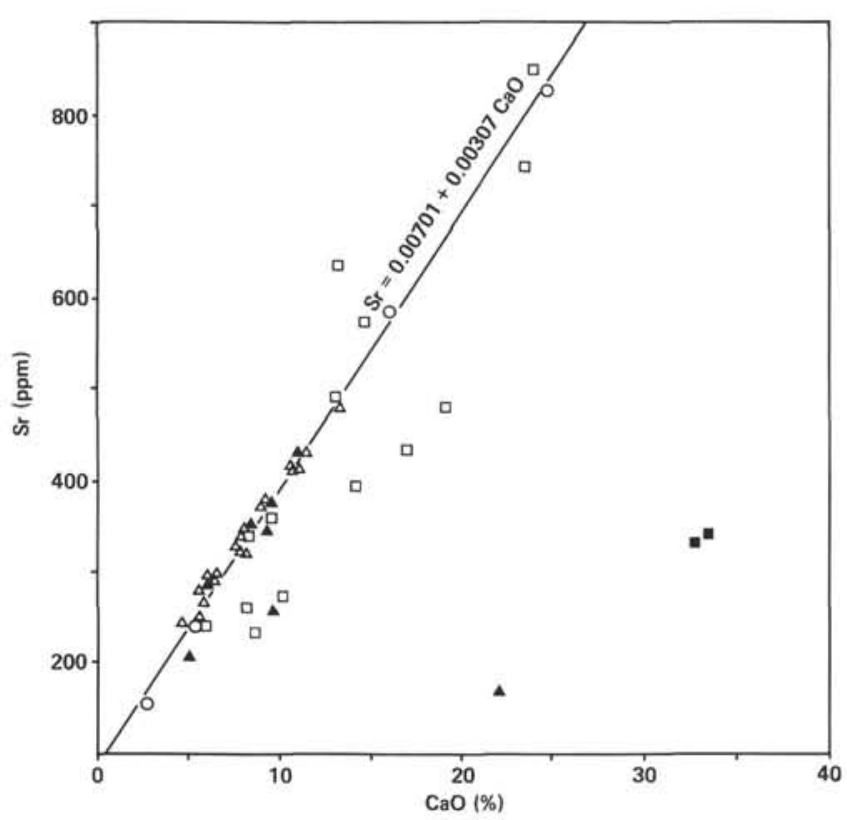

Figure 7. Correlation between the contents of $\mathrm{Sr}$ and $\mathrm{CaO}$ in the untreated total sediment samples. (Four calcitic samples represent marked positive correlation. Key for symbols is located in Fig. 6.)

\section{Fe and Mn}

Fe and $\mathrm{Mn}$, which are typical nonlithogenous elements in the deep-sea pelagic sediments (e.g., Chester and Hughes, 1967; Chester et al., 1967; Krishnaswami, 1967), tend to concentrate in sediments initially as $\mathrm{Fe}$ and Mn oxides from overlying seawater through an hydrogenous process, and often form ferromanganese nodules. The $\mathrm{Fe}_{2} \mathrm{O}_{3}$ content in total samples at Site 533 varies between $1.65 \%$ in dolomitic lenses (Section 533A$5-1$ ) and $10.6 \%$ in siderite-rich mud (Section 533A-184), averaging $5.8 \%$. The highest content of $\mathrm{MnO}$ was also detected in Section 533A-18-4, but the lowest value is found in sparsely calcareous mud (Section 533-39-3). Variation of $\mathrm{Fe}_{2} \mathrm{O}_{3}$ and $\mathrm{MnO}$ with depth is shown in Figures 8 and 9 . The contribution of soluble fraction $\left(R_{\mathrm{SF}}\right)$ is also given in these figures.

\section{$\mathrm{Fe}_{2} \mathrm{O}_{3}$}

The $\mathrm{Fe}_{2} \mathrm{O}_{3}$ content in total samples is higher in the Pliocene sediments $(6.35 \%$ on an average) than in the Holocene-Pleistocene sediments $(5.09 \%$ on an average). This difference is due to soluble iron. The fluctuation in total iron content is subparallel to the fluctuation of soluble iron content $\left(C_{\mathrm{SF}}\right)$, as shown in Fig. 8 . The soluble iron is considered to be contained chiefly as siderite and ferromanganese oxide, because calcite and dolomite are poor in iron, as discussed in the next section. The contribution of soluble iron $\left(R_{\mathrm{SF}}\right)$ seems to increase with depth as 0 to $20 \%$ in the Pleistocene sediments and 10 to $30 \%$ in the Pliocene sediments. The higher content of total iron and the higher contribution of soluble iron in the Pliocene sediments seem to coincide with the occurrence of siderite. The highest content of soluble iron was found in the most sideritic section (533A-18-4). However, Section 533-28-3, which shows the second highest peak in Figure 8, does not contain siderite. This strongly suggests that significant amounts of soluble iron are present in the form of ferromanganese oxide.

\section{$\mathrm{MnO}$}

Total concentration $\left(C_{\mathrm{T}}\right)$ of $\mathrm{MnO}$ fluctuates greatly throughout the cores, particularly in the Pliocene sediments, due to variable contents of soluble manganese (much as does iron, Fig. 9). The MnO content in the insoluble residue $\left(C_{\mathrm{IR}}\right)$ varies little, averaging $0.065 \%$ for the Pleistocene and $0.049 \%$ for the Pliocene sediments. The contribution of soluble manganese $\left(R_{\mathrm{SF}}\right)$ is far greater than iron; the percentage of soluble MnO seems to increase with depth, ranging between 38 and $66 \%$ in the Pleistocene sediments and between 43 and $95 \%$ in the Pliocene sediments. In the deeper cores below 250 $\mathrm{m}$, values concentrate around $80 \%$. Extremely high total values and high contributions of soluble $\mathrm{MnO}$ are encountered in Sections 533A-9-3 and 533A-18-4. In the latter section, high $\mathrm{MnO}$ content can be attributed to the high content of siderite. In the former sections, however, the contents of soluble iron and siderite are not so high as to explain such high values for $\mathrm{MnO}$, and furthermore, Mn-carbonate was not detected in this sample. Therefore the soluble manganese in this sample is probably contained as $\mathrm{Mn}$ oxide.

\section{$\mathrm{MnO} / \mathrm{Fe}_{2} \mathrm{O}_{3}$ Ratio}

The ratios of $\mathrm{MnO}$ to $\mathrm{Fe}_{2} \mathrm{O}_{3}$ in total samples, insoluble residues, and in soluble fractions are shown in Figure 10. The ratios in the insoluble residues fall between 0.006 and 0.01 for most samples; they are somewhat 


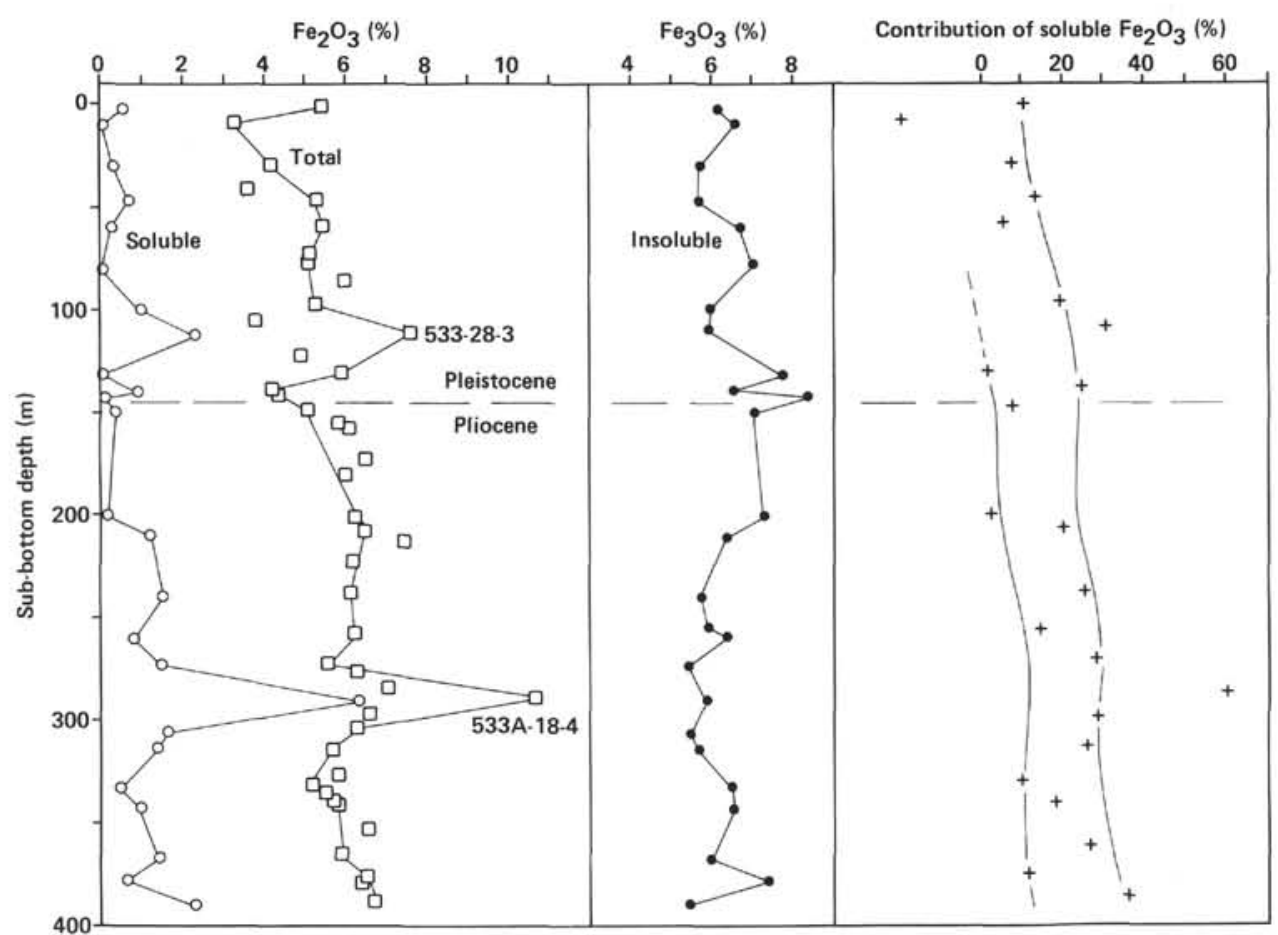

Figure 8. The stratigraphic variations in the contents of the total and soluble iron in the untreated samples (first column) and the contents in the insoluble residues (second column). (The contribution of soluble iron in the total sediment samples appears to increase with increasing depth [last column].)

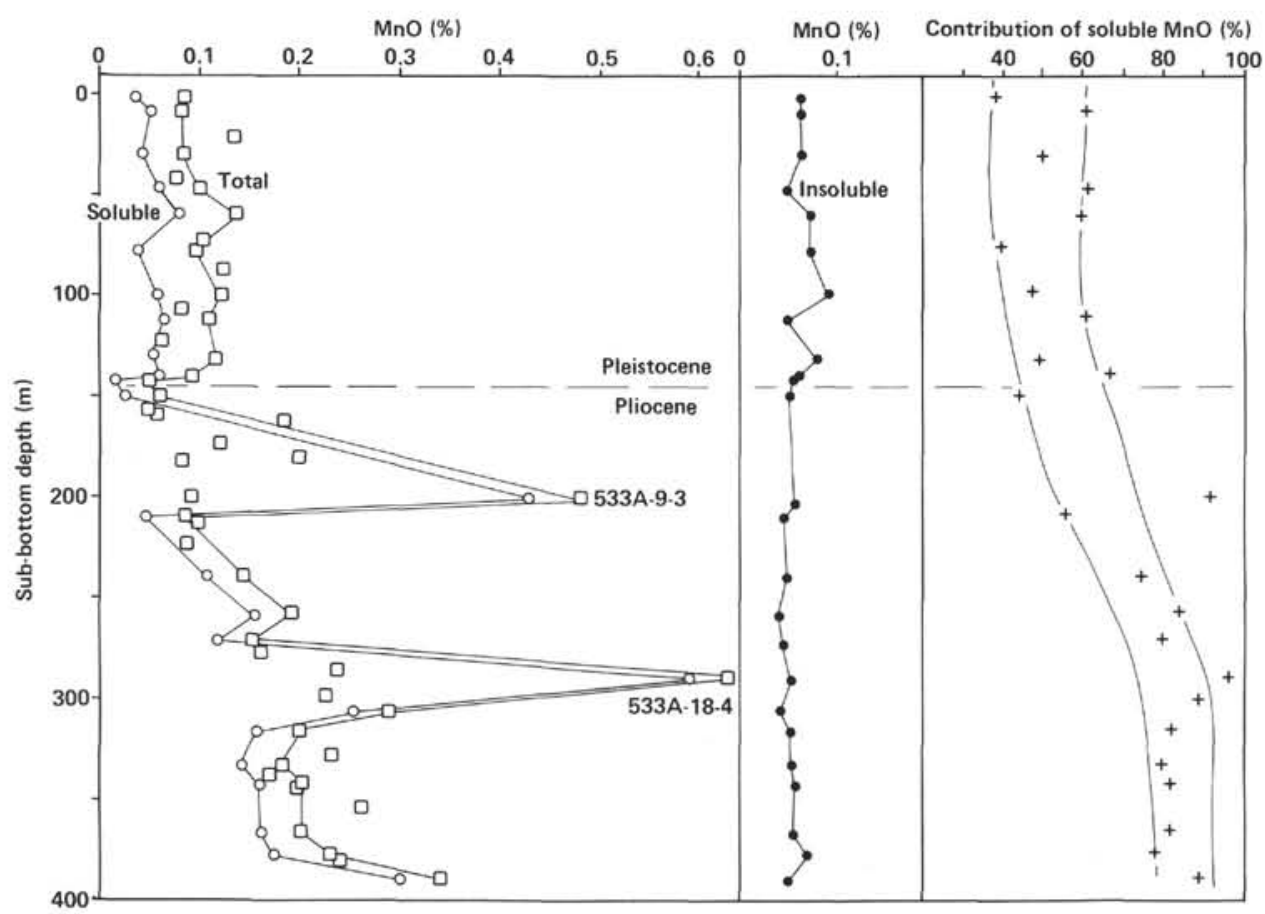

Figure 9. The stratigraphic variations in the contents of the total and soluble manganese in the untreated samples (first column) and the contents in the insoluble residues (second column). (The contribution of soluble manganese in the total sediment samples is remarkably high in cores below $200 \mathrm{~m}$ sub-bottom depth [last column].) 


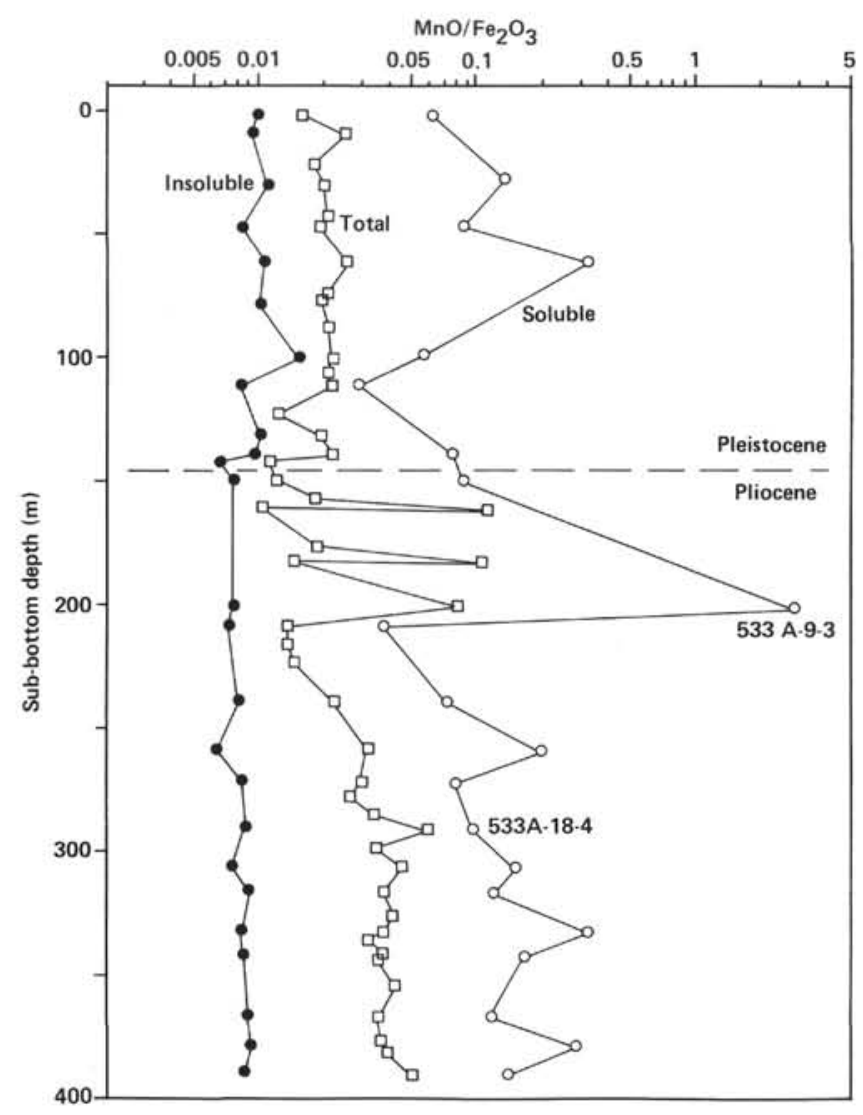

Figure 10. Variations of the $\mathrm{MnO} / \mathrm{Fe}_{2} \mathrm{O}_{3}$ ratio in the untreated total sediment samples, insoluble residues, and in the soluble fractions. (The ratio in the soluble fraction shows great fluctuation in the shallower cores, whereas it seems to converge toward the deeper cores.)

higher in the Pleistocene sediments, probably reflecting the different source of terrigenous sediments. As to total samples, ratios mostly range between 0.01 and 0.05 and are clearly higher in the Pliocene sediments due to the higher content of soluble manganese.

The ratio in the soluble fraction is widely scattered in the shallower cores, whereas it tends to concentrate in a narrow range in the deeper cores. Provided that the soluble iron and manganese in Section 533A-18-4 are chiefly from siderite, the $\mathrm{MnO} / \mathrm{Fe}_{2} \mathrm{O}_{3}$ ratio in the soluble fraction of this sample, 0.094 , is regarded as the ratio of siderite. Siderite in the Site 533 sediments is expected to have rather constant chemical composition, considering the relatively small fluctuation of $d_{104}$ spacing. Therefore, the deviation from the value 0.094 can be explained as due to variable content and variable composition of ferromanganese oxide. The large deviation in the shallow cores and convergence toward the deeper cores suggest that the ferromanganese oxide, of various chemical composition, is abundant in the shallow levels and reduced and precipitated as siderite in the deeper levels.

\section{Elements in Insoluble to Poorly Soluble Components}

Elements in the insoluble to poorly soluble components include $\mathrm{Ti}, \mathrm{K}, \mathrm{Ba}, \mathrm{Co}, \mathrm{Cu}, \mathrm{Ni}, \mathrm{Rb}$, and $\mathrm{Zn}$; con- tributions of soluble fractions $\left(R_{\mathrm{SF}}\right)$ are so small that these elements are considered to be contained mostly in the insoluble terrigenous suites. $\mathrm{Si}, \mathrm{Al}$, and $\mathrm{Na}$ should probably be included in this group as they are major components of aluminosilicates.

The abundances of these elements widely fluctuate in the Pleistocene sediments: the average concentrations are higher in the Pliocene sediments (Table 3). This fluctuation complements the variation of the carbonate content in the sediments; the fluctuation of the abundance of these elements was caused by the various degree of dilution of terrigenous materials by carbonates. In fact, the $\mathrm{SiO}_{2}$ content in total samples fluctuates widely, particularly in the Pleistocene sediments, whereas that in the insoluble residues concentrates around $65 \%$ with small deviation, as shown in Figure 11 . This value $(65 \%)$ is greater than the average $\mathrm{SiO}_{2}$ content of the pelagic clay $(57 \%)$, but similar to that of the offshore mud $(67 \%)$.

The insoluble residues of the Pleistocene sediments have, as a whole, higher contents of $\mathrm{CaO}(1.35 \%$ average) and higher $\mathrm{SiO}_{2} / \mathrm{Al}_{2} \mathrm{O}_{3}$ ratios (3.75 average) than those of the Pliocene sediments $(0.69 \%$ and 3.35 , respectively). This contrast is consistent with the bulk $\mathrm{X}$-ray mineralogy results showing that the Pleistocene sediments abound in terrigenous components such as quartz and plagioclase.

Very low contributions of soluble $\mathrm{Ba}\left(\mathrm{R}_{\mathrm{SF}}=0.1-0.2\right)$ suggest that $\mathrm{Ba}$ is associated with non carbonate phases. Given the existence of a barite vein in Section 533-34-3, we conclude that $\mathrm{Ba}$ is probably present as sulfate.

\section{Comparison with Modern Sediments}

The abundances of selected elements on a carbonatefree basis were calculated from the total concentration

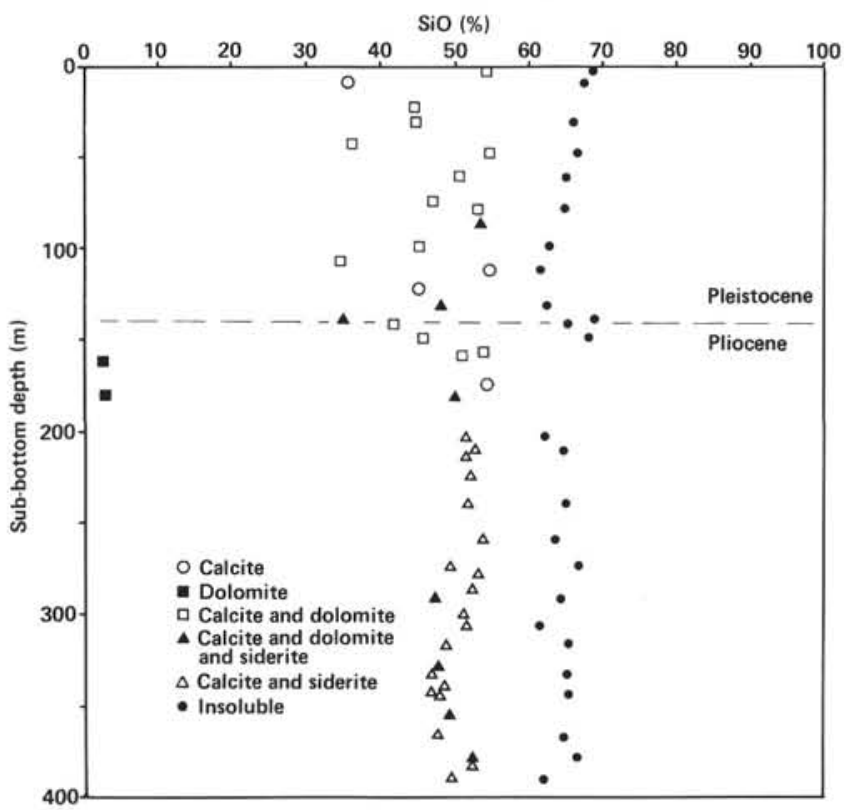

Figure 11. Fluctuation of the $\mathrm{SiO}_{2}$ content in the Site 533 sediments with depth. (The contents in the total sediment samples fluctuate widely, particularly in the Pliocene sediments, whereas those in the insoluble residues concentrate around 65 to $67 \%$.) 
$\left(C_{\mathrm{T}}\right)$ and the percentage of carbonate content $(X)$ in order to compare the base metal contents in the Site 533 sediments with those in the offshore mud and pelagic clay. Figure 12 shows the range and average of $\mathrm{Fe}_{2} \mathrm{O}_{3}$, $\mathrm{MnO}, \mathrm{V}, \mathrm{Cu}, \mathrm{Ni}$, and Co contents of the offshore mud, pelagic clay, and Site 533 sediments. $\mathrm{V}, \mathrm{Cu}, \mathrm{Ni}$, and $\mathrm{Co}$ are typical hydrogenous elements that tend to concentrate more in pelagic clay. However, at Site 533, the abundances of these elements are similar to or smaller than those in the offshore mud. This suggests that the Pliocene and Pleistocene sediments on the Blake Outer Ridge accumulated under strong influence of terrigenous influxes. The soluble hydrogenous portion of the base metals increases, as the sedimentation rate decreases (Krishnaswami, 1976; Matsumoto and Iijima, in press). Low contribution of soluble $\mathrm{Co}, \mathrm{Cu}$, and $\mathrm{Ni}$ at Site 533 probably reflect a rapid rate of sedimentation, 7 to $21 \mathrm{~cm} / 1000 \mathrm{yr}$., on the Blake Outer Ridge.

\section{Chemical Composition of Carbonate Minerals}

\section{Calcite}

Tests of foraminifers show nearly end-member composition of calcite, as shown in Table 4 and Figure 13. The contents of $\mathrm{MgCO}_{3}(0.41-0.42 \mathrm{~mol} \%)$ and $\mathrm{Sr}(1100$ $1300 \mathrm{ppm}$ ) are somewhat similar to the values estimated from the bulk chemistry of calcitic samples. $\mathrm{FeCO}_{3}$ and $\mathrm{MnCO}_{3}$ contents are lower than $0.3 \mathrm{~mol} \%$. Irregularly shaped authigenic(?) calcite, which appears to fill inter- stices, is also poor in $\mathrm{MgCO}_{3}(0.43-2.4 \mathrm{~mol} \%)$ and $\mathrm{FeCO}_{3}(0.21-0.76 \mathrm{~mol} \%)$.

\section{Dolomite}

Dolomite exhibits a composition ranging from calcian dolomite to ferroan dolomite through nearly endmember dolomite (Fig. 13). Weakly corroded rhombs scattered in sediments are poor in iron (0.1-0.8 mol\% $\mathrm{FeCO}_{3}$ ), with variable contents of magnesium (42.4$\left.49.4 \mathrm{~mol} \% \mathrm{MgCO}_{3}\right)$. In spite of wider compositional variation, a regular pattern of chemical zoning was not detected in these rhombs.

Interlocking polyhedral grains forming dolomitic lenses are characterized by higher contents of $\mathrm{FeCO}_{3}(0.8-$ $2.7 \mathrm{~mol} \%)$ and $\mathrm{MnCO}_{3}(0.15-0.25 \% \mathrm{~mol} \%)$, compared with isolated rhombs. In contrast with $\mathrm{Fe}$ and $\mathrm{Mn}, \mathrm{Sr}$ is more concentrated in the isolated rhombs $(1500 \mathrm{ppm}$ average) than in the interlocking polyhedral grains ( 350 ppm average).

\section{Siderite}

Siderite is always a subordinate constituent of carbonate sediments at Site 533 and usually occurs as rhombs so fine and friable that XMA analysis could not be satisfactorily accomplished. Chemical composition of siderite can be estimated from the bulk chemical composition on the assumption that all the soluble $\mathrm{Fe}$ and $\mathrm{Mn}$ in Section 533A-18-4 is derived from siderite: the contents of soluble $\mathrm{Fe}_{2} \mathrm{O}_{3}$ and $\mathrm{MnO}$ are $6.29 \%$ and

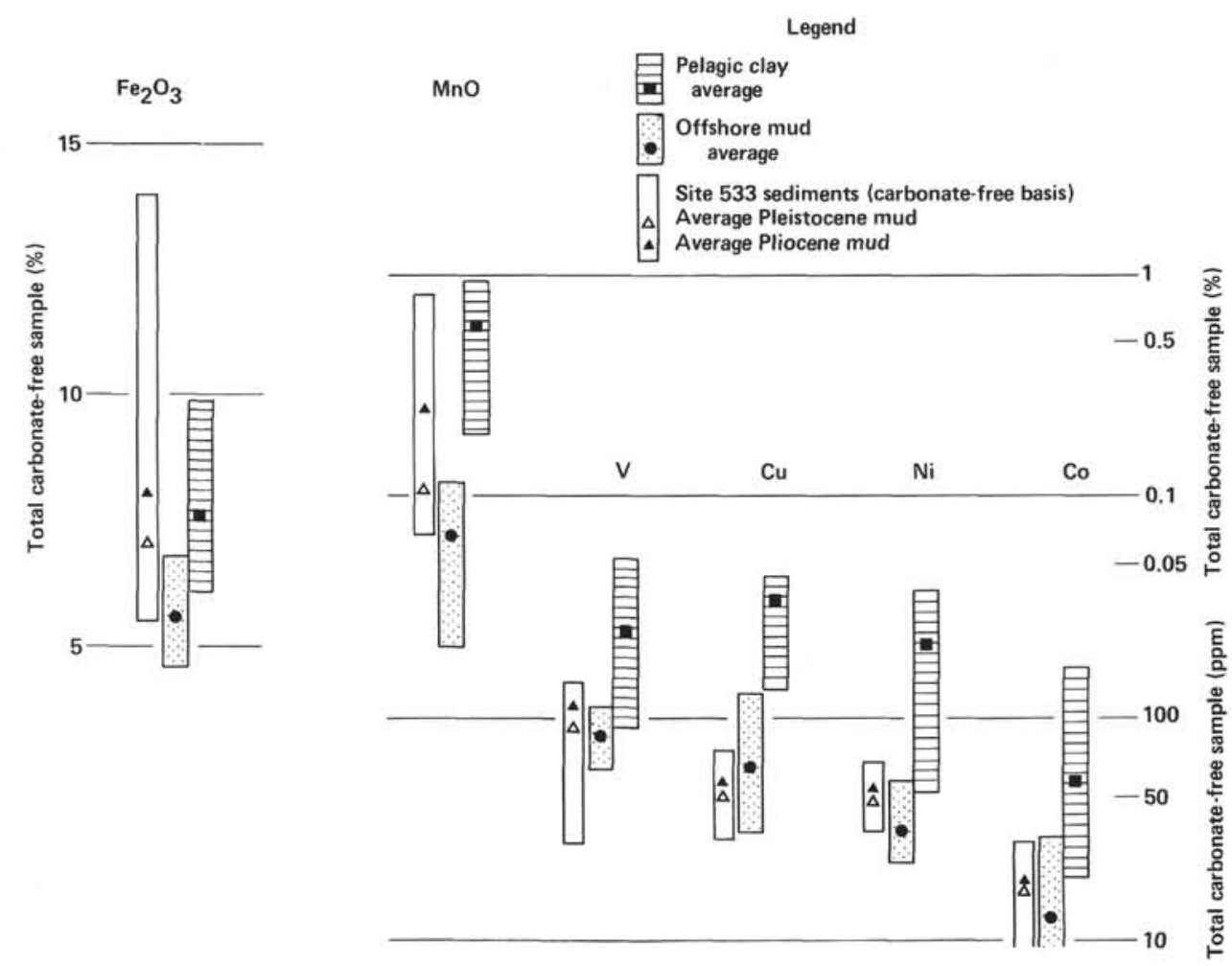

Figure 12. Range and average of total iron as $\mathrm{Fe}_{2} \mathrm{O}_{3}, \mathrm{MnO}, \mathrm{V}, \mathrm{Cu}, \mathrm{Ni}$, and Co contents in the pelagic clay, offshore mud, and Site 533 sediments on a carbonate-free basis.(Data on the recent sediments are from Matsumoto and lijima [in press].) 
Table 4. Chemical composition of calcite (cal.) and dolomite (dol.) in the Site 533 sediments.

\begin{tabular}{|c|c|c|c|c|c|c|c|c|c|c|c|c|c|c|c|c|c|c|c|c|c|c|c|}
\hline \multirow{2}{*}{$\begin{array}{l}\text { Section } \\
\text { Analysis no. } \\
\text { Carbonates }\end{array}$} & \multicolumn{6}{|c|}{$533 A-18-4$} & \multicolumn{5}{|c|}{$533-34-3$} & \multicolumn{6}{|c|}{$533 A-5-1$} & \multicolumn{6}{|c|}{$533 A-7-1$} \\
\hline & $\begin{array}{c}1 \\
\mathrm{cal} .\end{array}$ & $\underset{\mathrm{cal}}{2}$ & $\begin{array}{c}3 \\
\text { cal. }\end{array}$ & $\begin{array}{c}4 \\
\text { cal. }\end{array}$ & $\begin{array}{c}5 \\
\text { cal. }\end{array}$ & $\begin{array}{c}6 \\
\text { cal. }\end{array}$ & $\begin{array}{c}7 \\
\text { dol. }\end{array}$ & $\begin{array}{c}8 \\
\text { dol. }\end{array}$ & $\begin{array}{c}9 \\
\text { dol. }\end{array}$ & $\begin{array}{r}10 \\
\text { dol. }\end{array}$ & $\begin{array}{c}11 \\
\text { dol. }\end{array}$ & $\begin{array}{c}12 \\
\text { dol. }\end{array}$ & $\begin{array}{r}13 \\
\text { dol. }\end{array}$ & $\begin{array}{c}14 \\
\text { dol. }\end{array}$ & $\begin{array}{c}15 \\
\text { dol. }\end{array}$ & $\begin{array}{c}16 \\
\text { dol. }\end{array}$ & $\begin{array}{c}17 \\
\text { dol. }\end{array}$ & $\begin{array}{c}18 \\
\text { dol. }\end{array}$ & $\begin{array}{r}19 \\
\text { dol. }\end{array}$ & $\begin{array}{c}20 \\
\text { dol. }\end{array}$ & $\begin{array}{c}21 \\
\text { dol. }\end{array}$ & $\begin{array}{c}22 \\
\text { dol. }\end{array}$ & $\begin{array}{r}23 \\
\text { dol. }\end{array}$ \\
\hline $\mathrm{MgO}$ & 0.16 & 0.16 & 0.57 & 0.95 & 0.24 & 0.17 & 1.27 & 21.71 & 22.32 & 20.74 & 20.69 & 18.87 & 18.21 & 19.27 & 17.63 & 20.01 & 17.36 & 19.63 & 19.26 & 19.67 & 18.75 & 19.10 & 18.90 \\
\hline $\mathrm{CaO}$ & 53.15 & 53.90 & 55.38 & 54.70 & 53.99 & 54.45 & 31.42 & 32.26 & 31.54 & 31.50 & 31.84 & 30.72 & 27.98 & 31.80 & 29.07 & 31.85 & 27.73 & 30.23 & 28.91 & 29.72 & 28.12 & 29.58 & 29.14 \\
\hline $\mathrm{MnO}$ & 0.01 & 0.18 & 0.03 & - & - & - & 0.0 & 0.0 & 0.02 & 0.0 & 0.0 & 0.17 & 0.14 & 0.16 & 0.17 & 0.16 & 0.14 & 0.11 & 0.15 & 0.16 & 0.12 & 0.14 & 0.14 \\
\hline $\mathrm{FeO} \cdot$ & 0.0 & 0.17 & 0.15 & 0.19 & 0.53 & 0.15 & 0.09 & 0.09 & 0.11 & 0.08 & 0.07 & 1.43 & 1.60 & 0.81 & 1.88 & 0.64 & 1.51 & 1.02 & 1.01 & 1.39 & 1.27 & 0.95 & 1.65 \\
\hline SrO & 0.13 & 0.15 & 0.37 & - & - & - & 0.18 & 0.0 & 0.20 & 0.0 & 0.13 & 0.0 & 0.0 & 0.09 & 0.0 & 0.0 & 0.0 & 0.13 & 0.0 & 0.04 & 0.0 & 0.0 & 0.24 \\
\hline $\mathrm{CO}_{2}$ & 41.93 & 42.75 & 44.35 & 44.07 & 42.95 & 43.00 & 48.03 & 49.06 & 49.31 & 47.40 & 47.69 & 45.68 & 42.89 & 46.63 & 43.31 & 47.32 & 41.72 & 45.91 & 44.42 & 45.77 & 43.38 & 44.73 & 44.74 \\
\hline $\mathrm{MgCO}_{3}$ & 0.41 & 0.42 & 1.40 & 2.35 & 0.62 & 0.43 & 48.33 & 48.31 & 49.41 & 47.75 & 47.36 & 45.08 & 46.34 & 45.11 & 44.43 & 46.16 & 45.42 & 46.67 & 47.33 & 46.93 & 47.18 & 46.62 & 46.11 \\
\hline $\mathrm{CaCO}_{3}$ & 99.44 & 98.93 & 97.99 & 97.38 & 98.67 & 99.35 & 51.32 & 51.58 & 50.18 & 52.14 & 52.39 & 52.77 & 51.17 & 53.50 & 52.68 & 52.81 & 52.15 & 51.66 & 51.07 & 50.95 & 50.85 & 51.89 & S1.11 \\
\hline $\mathrm{MnCO}_{3}$ & 0.02 & 0.26 & 0.05 & - & - & - & 0.0 & 0.0 & 0.02 & 0.0 & 0.0 & 0.24 & 0.20 & 0.21 & 0.24 & 0.21 & 0.20 & 0.15 & 0.20 & 0.21 & 0.17 & 0.19 & 0.20 \\
\hline $\mathrm{FeCO}_{3}$ & 0.0 & 0.25 & 0.21 & 0.27 & 0.76 & 0.22 & 0.11 & 0.11 & 0.14 & 0.10 & 0.0 & 1.92 & 2.29 & 1.06 & 2.66 & 0.82 & 2.22 & 1.36 & 1.39 & 1.86 & 1.79 & 1.30 & 2.25 \\
\hline $\mathrm{SrCO}_{3}$ & 0.13 & 0.15 & 0.36 & - & - & - & 0.16 & 0.0 & 0.18 & 0.0 & 0.11 & 0.0 & 0.0 & 0.80 & 0.0 & 0.0 & 0.0 & 0.11 & 0.0 & 0.03 & 0.0 & 0.0 & 0.23 \\
\hline
\end{tabular}

Note: $\mathrm{FeO}^{*}=$ total iron as $\mathrm{FeO}:-=$ not determined; analysis nos. $1,2=$ foraminiferal test, nos. $3-6=$ authigenic calcite, nos. $7-11=$ isolated corroded rhombs, nos. $12-23=$ interlocking polyhedrons. nos. $24-47=$ isolated corroded rhombs.

$0.59 \%$, respectively. The soluble $\mathrm{MgO}$ content is estimated to be around $1.5 \%$ from Figure 6 . Hence, the mole ratio of $\mathrm{Fe}, \mathrm{Mg}$, and $\mathrm{Mn}$ is 63:30:7. The $\mathrm{CaCO}_{3}$ content is obtained from the $d_{104}$ spacing ( $2.794 \AA$ ) and the mole ratio of $\mathrm{Fe}+\mathrm{Mn}: \mathrm{Mg}$, using the diagram prepared by Matsumoto et al. (1978). The estimated composition is $\left(\mathrm{Fe}_{0.60} \mathrm{Mg}_{0.32} \mathrm{Ca}_{0.07} \mathrm{Mn}_{0.01}\right) \mathrm{CO}_{3}$, as shown in Figure 13. This composition is quite similar to that of the deep-sea siderite from Site 439 off northeastern Japan (Matsumoto and Iijima, 1980) and falls within the range of shallow marine siderite from the Paleogene strata of Japan (Matsumoto, 1978; Matsumoto and Iijima, 1981).

\section{DISCUSSION}

\section{Diagenetic Zonation of Carbonates}

Based on the distribution of authigenic dolomite and siderite, three diagenetic zones of carbonates are set up in the Holocene to the Pliocene sections at Site 533, as represented in Figure 14. Zone I (dolomite zone, Holocene to middle Pleistocene) corresponds to the common occurrence of dolomite without siderite; Zone II (transition zone, middle Pleistocene to upper Pliocene) contains a variable amount of dolomite with occasional siderite; Zone III (siderite zone, upper and middle Pliocene) is characterized by the common occurrence of siderite with sporadic, trace amounts of dolomite. Considering the fluctuation of $d_{104}$ spacing of dolomite and the results of XMA analysis, dolomite composition seems to change with depth from a calcian type in Zones I and II to a ferroan type in Zone II. Authigenic pyrite appears throughout Zones I, II, and III. Clinoptilolite occurs exclusively in Zones II and III, although it appears infrequently.

\section{Zone I (dolomite zone)}

Site 533 sediments are rich in organic substrate; the organic carbon levels are around 0.5 to $1.0 \%$ (Site 533 report, this volume). The oxidation of organic carbon by aerobic organisms is, in general, limited to surficial sediments; probably less than $10 \mathrm{~cm}$ (Irwin et al., 1977). After exhaustion of dissolved oxygen in the pore water, organic matter is decomposed by anaerobic bacteria using $\mathrm{SO}_{4}{ }^{2-}, \mathrm{NO}_{3}{ }^{2-}$, or oxydized components as oxidizing agents (Berner, 1971). Sulfate reduction produces carbonate species along with sulfide:

$$
2 \mathrm{CH}_{2} \mathrm{O}+\mathrm{SO}_{4}{ }^{2-} \rightarrow 2 \mathrm{HCO}_{3}^{-}+\mathrm{H}_{2} \mathrm{~S} \text {. }
$$

In the sediments poor in dissolved oxygen, ferromanganese oxide is reduced to ferrous iron. $\mathrm{Fe}^{2+}$ in the pore water immediately precipitates as pyrite because of the very low solubility of iron sulfide within a few tens of meters depth, where sulfate reduction occurs actively. The amount of ferromanganese oxide that escaped from pyritization in Zone I is principally controlled by the amount of sulfide species generated through sulfate reduction in the surficial sediments. Downward diffusion of sulfate from overlying seawater is probably low in the rapidly accumulated sediments (Curtis, 1977). Therefore at Site 533 the amount of pyrite is relatively small; significant amounts of ferromanganese oxide have survived and been reworked into deeper levels to form $\mathrm{Fe}$-carbonates at later stages.

High alkalinity due to sulfate reduction, high $\mathrm{pH}$ caused by nitrate reduction, and a high $\mathrm{Mg}^{2+} / \mathrm{Ca}^{2+}$ ratio in the pore water of the Site 533 sediments (Fig. 15) are all favorable for the precipitation of dolomite. The gradual decrease of $\mathrm{Mg}^{2+}$ in Zones I and II with increasing depth (Fig. 15) indicates that dolomitization continues into Zone II and stops there. As suggested in the preceding section, dolomite probably replaces precursor biogenic calcite. Low contents of $\mathrm{Fe}$ and $\mathrm{Mn}$ in dolomite of Zone I indicate the fact that $\mathrm{Fe}^{2+}$ and $\mathrm{Mn}^{2+}$ activities are maintained at very low levels due to the slow rate of reduction of ferromanganese oxide and/or due to $\mathrm{Fe}$. Mn-sulfide precipitation in this Zone.

\section{Zone II (transition zone)}

With increasing depth of burial, reduction of ferromanganese oxide is accelerated under high temperature and lower Eh (oxidation potential) conditions. As clearly indicated by Curtis (1977), the reduction of ferric iron is accompanied by equivalent oxidation of organic matter:

$$
2 \mathrm{Fe}_{2} \mathrm{O}_{3}+3 \mathrm{H}_{2} \mathrm{O}+\mathrm{CH}_{2} \mathrm{O} \rightarrow 4 \mathrm{Fe}^{2+}+\mathrm{HCO}_{3}{ }^{-}+70 \mathrm{H}^{-} \text {. }
$$

This reaction results in higher alkalinity and high $\mathrm{pH}$ as well as higher concentration of ferrous iron. On the other hand, sulfate reduction is not expected to continue into deeper levels due to the diminishing of sulfate species with depth. As soon as sulfate reduction ceases, bacterial fermentation of organic substrate $\left(2 \mathrm{CH}_{2} \mathrm{O} \rightarrow\right.$ 
Table 4. (Continued).

\begin{tabular}{|c|c|c|c|c|c|c|c|c|c|c|c|c|c|c|c|c|c|c|c|c|c|c|c|}
\hline \multicolumn{24}{|c|}{$533-18-4$} \\
\hline $\begin{array}{c}24 \\
\text { dol. }\end{array}$ & $\begin{array}{c}25 \\
\text { dol. }\end{array}$ & $\begin{array}{c}26 \\
\text { dol. }\end{array}$ & $\begin{array}{c}27 \\
\text { dol. }\end{array}$ & $\begin{array}{c}28 \\
\text { dol. }\end{array}$ & $\begin{array}{c}29 \\
\text { dol. }\end{array}$ & $\begin{array}{c}30 \\
\text { dol. }\end{array}$ & $\begin{array}{c}31 \\
\text { dol. }\end{array}$ & $\begin{array}{c}32 \\
\text { dol. }\end{array}$ & $\begin{array}{c}33 \\
\text { dol. }\end{array}$ & $\begin{array}{c}34 \\
\text { dol. }\end{array}$ & $\begin{array}{c}35 \\
\text { dol. }\end{array}$ & $\begin{array}{c}36 \\
\text { dol. }\end{array}$ & $\begin{array}{c}37 \\
\text { dol. }\end{array}$ & $\begin{array}{c}38 \\
\text { dol. }\end{array}$ & $\begin{array}{c}39 \\
\text { dol. }\end{array}$ & $\begin{array}{c}40 \\
\text { dol. }\end{array}$ & $\begin{array}{c}41 \\
\text { dol. }\end{array}$ & $\begin{array}{c}42 \\
\text { dol. }\end{array}$ & $\begin{array}{c}43 \\
\text { dol. }\end{array}$ & $\begin{array}{c}44 \\
\text { dol. }\end{array}$ & $\begin{array}{c}45 \\
\text { dol. }\end{array}$ & $\begin{array}{l}46 \\
\text { dol. }\end{array}$ & $\begin{array}{l}47 \\
\text { dol. }\end{array}$ \\
\hline 18.96 & 19.44 & 19.59 & 19.16 & 18.79 & 18.83 & 19.31 & 18.89 & 18.84 & 19.03 & 19.18 & 18.52 & 17.78 & 19.36 & 18.92 & 20.49 & 20.44 & 18.47 & 20.84 & 19.74 & 18.21 & 18.41 & 18.48 & 18.31 \\
\hline 33.60 & 33.57 & 33.75 & 33.22 & 33.46 & 30.42 & 30.42 & 30.96 & 33.35 & 32.77 & 32.57 & 32.14 & 33.42 & 32.59 & 31.35 & 32.54 & 33.50 & 33.27 & 29.75 & 31.99 & 32.74 & 33.15 & 34.40 & 34.15 \\
\hline 0.11 & 0.01 & 0.0 & 0.02 & 0.0 & 0.02 & 0.02 & 0.07 & 0.0 & 0.0 & 0.0 & 0.18 & 0.0 & 0.0 & - & - & - & - & - & - & - & - & - & - \\
\hline 0.11 & 0.07 & 0.08 & 0.08 & 0.09 & 0.37 & 0.57 & 0.45 & 0.05 & 0.10 & 0.08 & 0.29 & 0.17 & 0.16 & 0.20 & 0.61 & 0.14 & 0.12 & 0.38 & 0.18 & 0.19 & 0.20 & 0.11 & 0.14 \\
\hline 0.0 & 0.36 & 0.32 & 0.10 & 0.12 & 0.28 & 0.41 & 0.19 & 0.07 & 0.22 & 0.55 & 0.27 & 0.0 & 0.0 & - & - & - & - & - & - & - & - & - & - \\
\hline 47.26 & 47.82 & 48.10 & 47.09 & 46.88 & 44.83 & 45.55 & 45.44 & 46.79 & 46.67 & 46.88 & 45.89 & 45.73 & 46.80 & 45.37 & 48.27 & 48.68 & 46.34 & 46.32 & 46.77 & 45.67 & 40.23 & 47.22 & 46.86 \\
\hline 43.78 & 44.37 & 44.45 & 44.40 & 43.74 & 45.85 & 46.26 & 45.60 & 42.96 & 44.50 & 44.66 & 44.07 & 42.44 & 45.15 & 45.52 & 46.33 & 45.83 & 43.51 & 49.10 & 46.09 & 43.51 & 43.48 & 42.71 & 42.64 \\
\hline 55.79 & 55.08 & 55.04 & 55.34 & 55.99 & 52.23 & 52.40 & 53.46 & 55.92 & 55.08 & 54.51 & 54.96 & 57.34 & 54.64 & 54.20 & 52.90 & 53.99 & 56.33 & 50.39 & 53.68 & 56.23 & 56.20 & 57.15 & 57.18 \\
\hline 0.28 & 0.01 & 0.0 & 0.02 & 0.0 & 0.02 & 0.02 & 0.09 & 0.0 & 0.0 & 0.0 & 0.24 & - & 0.0 & - & - & - & - & - & - & - & - & - & - \\
\hline 0.15 & 0.09 & 0.10 & 0.10 & 0.11 & 0.51 & 0.77 & 0.60 & 0.06 & 0.13 & 0.11 & 0.38 & 0.23 & 0.21 & 0.28 & 0.77 & 0.18 & 0.16 & 0.50 & 0.24 & 0.25 & 0.26 & 0.14 & 0.18 \\
\hline 0.0 & 0.31 & 0.28 & 0.09 & 0.10 & 0.27 & 0.39 & 0.18 & 0.06 & 0.20 & 0.50 & 0.25 & - & 0.0 & - & - & - & - & - & - & - & - & - & - \\
\hline
\end{tabular}

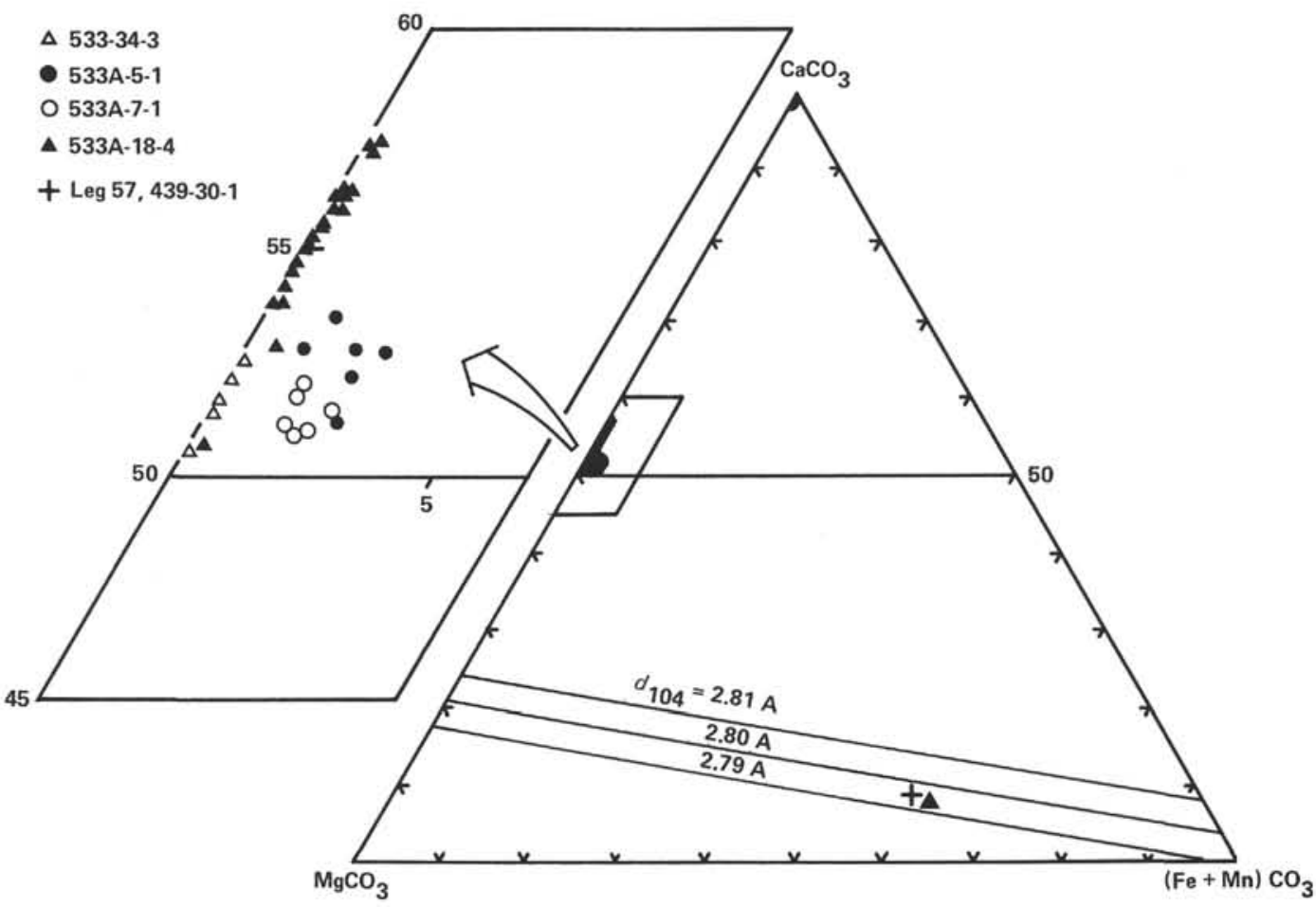

Figure 13. Chemical composition of calcite, dolomite, and siderite. (The composition of siderite in Section 533A-18-4 was estimated from the bulk chemical composition [see text], and that in Section 439-30-1, Leg 57 off northeastern Japan, was obtained by XMA [Matsumoto and Iijima, 1981].)

$\mathrm{CH}_{4}+\mathrm{CO}_{2}$ ) becomes the major reaction that decomposes organic matter and produces carbonate species. Under these conditions, iron carbonates are expected to precipitate in place of iron sulfide. An increase of the ratio $\mathrm{Fe}^{2+} / \mathrm{Ca}^{2+}$ in the pore water in Zone II has caused the precipitation of ferroan dolomite first, and then siderite.

The Fe content in dolomite is controlled by the pore water chemistry and the temperature (or approximately the burial depth) at which the coexisting siderite starts to precipitate (Smythe and Dunham, 1947). In fact, at Site 439 off northeastern Japan, magnesian siderite appears first at around $1080 \mathrm{~m}$ sub-bottom depth, and coexisting dolomite has an $(\mathrm{Fe}+\mathrm{Mn}) \mathrm{CO}_{3}$ component as high as $15 \mathrm{~mol} \%$ (Matsumoto and Iijima, 1980). At Site 533, magnesian siderite occurs in depths as shallow as $87 \mathrm{~m}$ sub-bottom, and ferroan dolomite contains only 3 $\mathrm{mol} \%(\mathrm{Fe}+\mathrm{Mn}) \mathrm{CO}_{3}$. Carbonate sediments at Site 533 are rich in iron and organic matter; they were deposited very rapidly, which impeded the reduction of ferric oxide and weakened the pyritization in the surficial sulfate reduction zone. These factors have probably brought about a rapid increase in the concentration of $\mathrm{Fe}^{2+}$; the $\mathrm{Fe}^{2+} / \mathrm{Ca}^{2+}$ ratio would have become high enough to precipitate siderite in place of ferroan dolomite at shallow depths. The estimated temperature of the first appearance of siderite is arount $6^{\circ} \mathrm{C}$.

\section{Zone III (siderite zone)}

Considering the general increase of $\mathrm{Fe}^{2+}$ in the pore water with burial depth, dolomite formed in Zone III is expected to have a higher content of iron compared with that of ferroan dolomite in Zone II. However, as shown in Figure 13, rhombohedral dolomite sparsely disseminated in Section 533A-18-4 (Zone III) is very low in iron, with a variable content of excess calcium in spite of its close association with siderite. This may suggest that the dolomite in Zone III precipitated originally at 


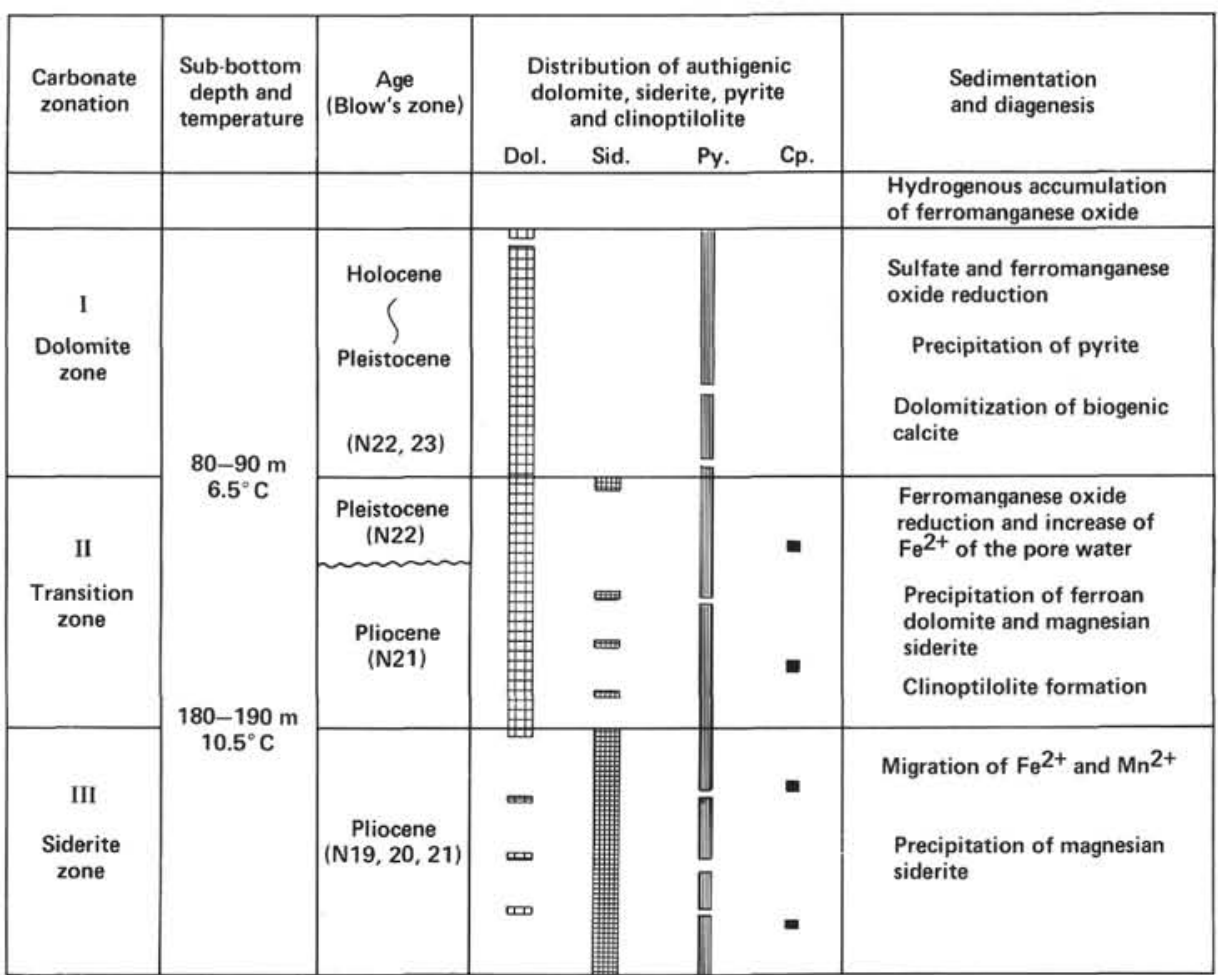

Figure 14. Zonation of carbonate diagenesis in the organic-matter-rich, rapidly accumulated sediments at Site 533 on the Blake Outer Ridge.

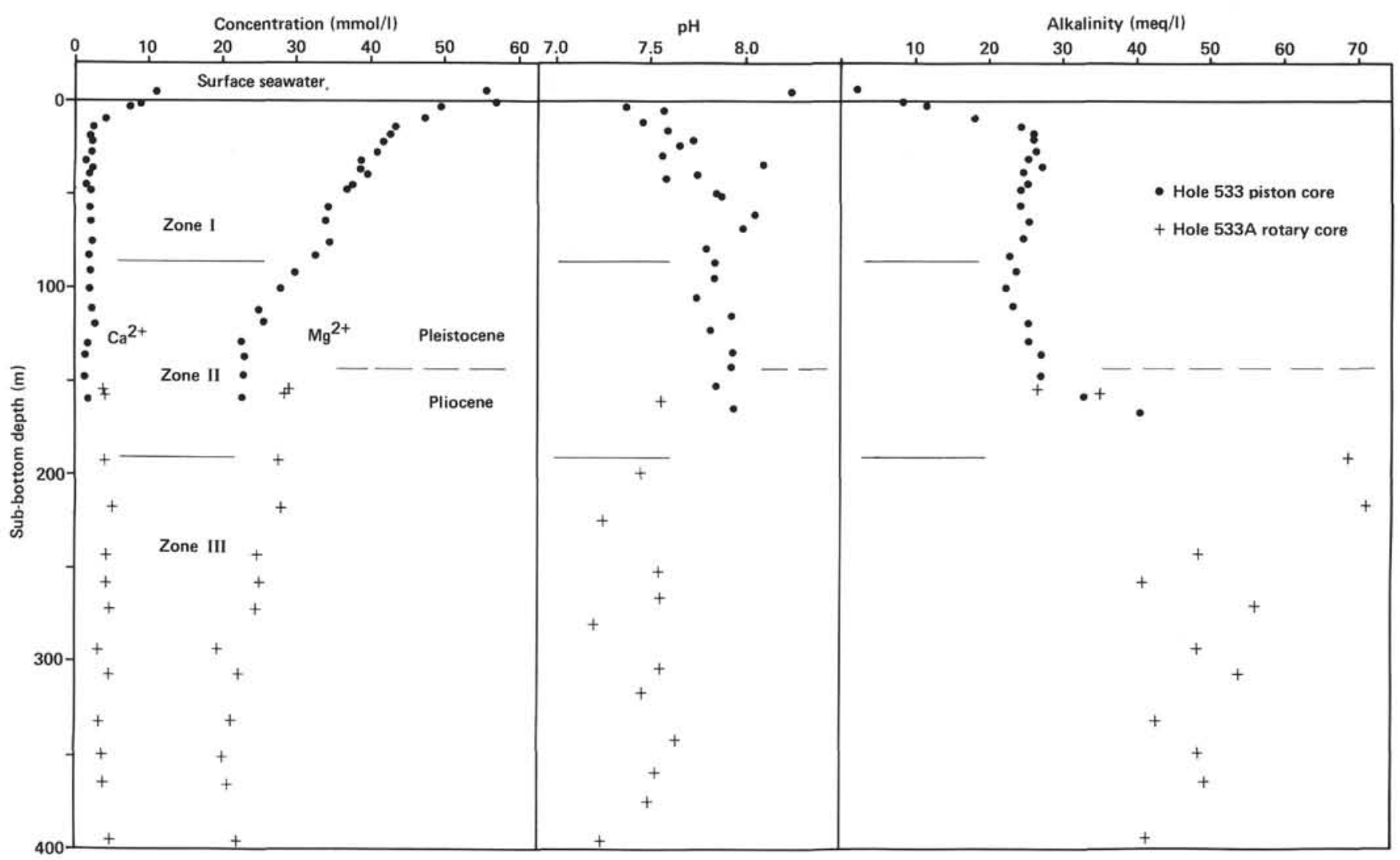

Figure 15. Chemical composition of the pore waters in the Site 533 sediments. (Mismatch of the chemical properties between overlapping portions of Holes 533 and $533 \mathrm{~A}$ appears to be attributable to a slight seawater contamination related to the switch from piston to rotary coring techniques [Sites 533 and 534 reports, this volume].) 
shallower levels. The scarcity of dolomite in Zone III can be explained as having been caused by dissolution or replacement by siderite; or alternatively, the Pliocene sediments were initially poor in dolomite due to originally high contents of $\mathrm{Fe}$ and $\mathrm{Mn}$. Corroded textures observed on the rhombs of low iron dolomite (Fig. 4B) and overgrowth of siderite on a dolomite rhomb observed at Site 102 (Lancelot and Ewing, 1972) favor the former explanation.

It is remarkable that Zone III apparently corresponds to the Pliocene sediments with high iron and manganese. The abundance of hydrogenous elements such as $\mathrm{Co}, \mathrm{Ni}, \mathrm{Cu}$, and $\mathrm{V}$ of the Pliocene sediments is low and falls within the range of the offshore mud, however, the $\mathrm{Fe}$ and $\mathrm{Mn}$ contents are extraordinarily high in spite of the rapid sedimentation (Fig. 12). Greater variation of the $\mathrm{Mn} / \mathrm{Fe}$ ratio in the soluble fraction in Zones I and II appears to converge downward in Zone III (Fig. 10). These facts favor the explanation that $\mathrm{Fe}$ and $\mathrm{Mn}$ migrated on a large scale in Zone III along with sideritization, and averaged the $\mathrm{Mn} / \mathrm{Fe}$ ratio in soluble fraction. However, a few samples contain markedly high contents of $\mathrm{Fe}$ and $\mathrm{Mn}$ as oxides, suggesting that the sediments were originally high in leacheable $\mathrm{Fe}$ and $\mathrm{Mn}$. Originally high content of iron explains the occurrence of siderite at shallow depths, as discussed before. Therefore it is concluded that both the originally high contents of $\mathrm{Fe}$ and $\mathrm{Mn}$ and the migration of $\mathrm{Fe}^{2+}$ and $\mathrm{Mn}^{2+}$ during sideritization are responsible for the high $\mathrm{Fe}$ and Mn of the Zone III sediments. Reworking of slowly accumulated pelagic clay with high $\mathrm{Fe}$ and $\mathrm{Mn}$ and redeposition on the Blake Outer Ridge may explain the high $\mathrm{Fe}$ and $\mathrm{Mn}$ contents in the rapidly accumulated sediments. The additional iron and manganese in Zone III was probably derived from the sediments beneath the Zone by upwardly migrating pore water.

\section{Comparison of Carbonate Zonation on the Blake Outer Ridge}

Correlations of stratigraphy and diagenetic zonation at Site 533 with the other DSDP sites on the Blake Outer Ridge are shown in a schematic cross-section in Figure
16. Data for Sites 102 and 103 are taken from Lancelot and Ewing (1972), Sayles et al. (1972), Ewing and Hollister (1972), and Zemmels et al. (1972) in DSDP Initial Reports, Volume 11. Site 102 on the crest of the Ridge represents stratigraphic succession, thickness, and diagenetic zonation similar to those at Site 533: siderite appears first at $100 \mathrm{~m}$ sub-bottom depth (quite similar to the depth of the first appearance of siderite at Site 533) and continuously occurs in the deeper cores beneath 270 $\mathrm{m}$; dolomite is found in all cores above $310 \mathrm{~m}$ subbottom depth. Site 103 on the southwestern flank of the Ridge is lacking most of the Pleistocene and Pliocene sediments, which are observed only in Core 1; Site 103 sediments are mostly Miocene, indicating sediment bypassing or erosion by strong bottom currents (Ewing and Hollister, 1972). Carbonate zonation at Site 103 is also markedly different from that at Sites 533 and 102. Siderite even occurs in Core 1 at a shallow $0.78-\mathrm{m}$ subbottom depth and is encountered in all cores below 100 $\mathrm{m}$; however, the siderite in Core 1 is not considered to have formed at the present depth, because pyrite, rather than siderite, is expected to precipitate where the sulfate concentration is as high as $1.35 \%$. Dolomite is infrequently present in Site 103 sediments except for Core 2 , which contains dolomitic lenses at $47 \mathrm{~m}$ sub-bottom depth. Considering these occurrences of siderite and dolomite, the boundary between Zones II and III at Site 103 can be located around $70 \mathrm{~m}$. Thus the carbonate zonation on the Blake Outer Ridge is subparallel to the bedding plane rather than to the present seafloor (Fig. 16). Zone I and the most of Zone II at Site 103 were probably eroded out after the carbonate diagenesis was complete. The thickness removed is estimated to be 110 to $200 \mathrm{~m}$.

In contrast to the carbonate zonation, natural gas zonation is subparallel to the present seafloor, as documented by Lancelot and Ewing (1972); the dotted area in Figure 16 indicates the transition zone between a free gas zone and a clathrate (gas hydrate) zone. The gas hydrate transition zone remains parallel to the seafloor, because the change of the gas phases has occurred during erosion, and the boundary of the gas zonation deep-

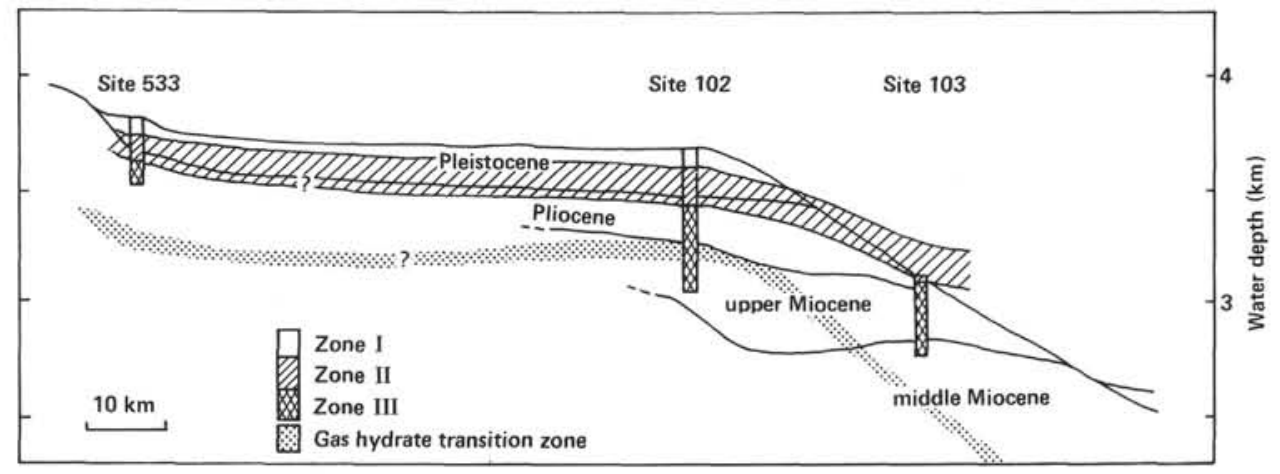

Figure 16. Schematic cross-section of the Blake Outer Ridge along the line indicated in Figure 1, showing the correlation of carbonate and gas zonations in Sites 533,102, and 103. (Gas zonation is parallel to the seafloor, but carbonate zonation is oblique to the seafloor in the flank region on the Ridge. At Site 103, Pleistocene and Pliocene sediments corresponding to Zones I and II were mostly eroded out by the strong bottom currents in the Pleistocene.) 
ened a few hundred meters, depending on the pressure and temperature after the removal of the overburden sediments.

Deep erosion on the flanks of the Ridge in the Pleistocene is probably related to the development of the Pleistocene glaciation, which apparently enhanced deep circulation. Distribution of clinoptilolite also indicates a large-scale erosion on the flank regions of the Ridge, as Iijima (1978) pointed out earlier.

The Blake Outer Ridge is believed to have formed through accretion of hemipelagic mud by sediment-laden bottom currents (e.g., Heezen et al., 1966; Ewing and Hollister, 1972). However, the present petrographi$\mathrm{cal}$ and geochemical study has revealed that deep subaqueous erosion was also the major agent in the construction of the Ridge; deep bottom currents have eroded and sculptured the Ridge, particularly in the northeastern and southwestern flank regions and formed the spitlike extension on the abyssal plain.

\section{CONCLUSION}

Organic-matter-rich, hemipelagic mud and clay from Site 533 on the Blake Outer Ridge contains various amounts of authigenic carbonates such as calcian dolomite, ferroan dolomite, and magnesian siderite, besides biogenic low magnesian calcite. Dolomite occurs largely in the shallow cores above $180 \mathrm{~m}$ sub-bottom depth, whereas siderite tends to concentrate in the cores below $190 \mathrm{~m}$.

Bulk geochemistry of the untreated total samples and the insoluble residues has revealed that the soluble fraction concentrates $\mathrm{Ca}, \mathrm{Mg}, \mathrm{Sr}, \mathrm{Mn}$, and $\mathrm{Fe}$, which are probably contained in carbonates and ferromanganese association. The typical hydrogenous elements such as $\mathrm{Co}, \mathrm{Cu}, \mathrm{Ni}$, and $\mathrm{V}$ in the Site 533 sediments occur in the insoluble group; the contents of these elements are very low, comparable to modern offshore mud, which probably reflects a high rate of sedimentation and high contribution of terrigenous suites at Site 533. The contents of $\mathrm{Fe}$ and $\mathrm{Mn}$, however, are very high for rapidly accumulated sediments, particularly those in the Pliocene mud. The high values are mainly due to the nonlithogenous soluble $\mathrm{Fe}$ and $\mathrm{Mn}$. The $\mathrm{Mn} / \mathrm{Fe}$ ratio in the soluble fraction fluctuates widely in the shallower cores, whereas it tends to converge in the deeper cores.

Based on the distribution of authigenic dolomite and siderite, diagenetic zonation of carbonates is defined as Zone I (dolomite zone, top-90 m), Zone II (transition zone, $90-180 \mathrm{~m}$ ), and Zone III (siderite zone, 180 m-bottom). In Zone I, bacterial reduction of sulfate from seawater and originally contained ferromanganese oxide brings about the precipitation of pyrite. The amount of ferric oxide converted to pyrite is small, because sulfate is rapidly exhausted in the quickly accumulated sediments. Under the high alkalinity, high $\mathrm{pH}$, and high $\mathrm{Mg}^{2+} / \mathrm{Ca}^{2+}$ ratio in the pore water, calcian dolomite precipitates in the form of isolated rhombohedrons, probably replacing biogenic calcite. In Zone II, increasing concentrations of ferrous iron in the pore water due to reduction of iron oxide caused the precipi- tation of ferroan dolomite and magnesian siderite. The shallow appearance of siderite at Site 533 is caused by the originally high contents of leacheable iron, abundant organic substrate, and rapid rate of sedimentation; these factors all favor the increase of the concentration of $\mathrm{Fe}^{2+}$ and carbonate species during burial. In Zone III, ferromanganese oxide is mostly reduced and fixed as siderite with increasing temperature. At least a part of iron in siderite is derived from the sediments beneath Zone III through upward migration of the pore water.

On the Blake Outer Ridge, Zones I, II, and III are all recognized in the crest regions (Sites 533 and 102), whereas Zone I and most of Zone II are not found in the flank regions (Site 103), because of the erosion of most Pleistocene and Pliocene sediments resulting from the enhanced deep circulation in the Pleistocene. The thickness eroded out is estimated to be 110 to $200 \mathrm{~m}$.

\section{ACKNOWLEDGMENTS}

I am grateful to the Leg 76 shipboard particiants, particularly Dr. H. Kagami (University of Tokyo), who collected the samples for this study. I thank Dr. S. Ohara (Rigaku Industrial Corporation) for EDX analyses and Mr. H. Hidaka (Hitachi, Ltd.) for SEM examination and analyses of carbonates using the EDAX system. Professors C. D. Curtis (Sheffield University) and A. Iijima (University of Tokyo) kindly reviewed the manuscript and offered helpful suggestions and comments. This research was financially supported by a Grant-in-Aid from the Ministry of Education of Japan (No. 56540463) and Ito Science Foundation.

\section{REFERENCES}

Berner, R. A. 1971. Principles of Chemical Sedimentology: New York (McGraw-Hill).

Chester, R., Aston, S. R., and Gruty, D., 1976. The trace-element partitioning geochemistry in an ancient deep-sea sediment core from the Bermuda Rise. Mar. Geol., 21:271-288.

Chester, R., and Hughes, M. J., 1967. A chemical technique for the separation of ferromanganese minerals, carbonate minerals, and adsorbed trace elements from pelagic sediments. Chem. Geol., 2; 249-263.

Chester, R., and Messina-Hanna, R. G., 1970. Trace element partition patterns in North Atlantic deep-sea sediments. Geochim. Cosmochim. Acta, 34:1121-1128.

Curtis, C. D., 1977. Sedimentary geochemistry: environments and processes dominated by involvement of aqueous phases. Philos. Trans. R. Soc. London, A286:353-372.

Ewing, J. I., and Hollister, C. D., 1972. Regional aspects of deep sea drilling in the western North Atlantic. In Hollister, C. D., and Ewing, J. I., et al., Init. Repts. DSDP, 11: Washington (U.S. Govt. Printing Office), 951-973.

Heezen, B. C., Hollister, C. D., and Riddiman, W. F., 1966. Shaping of the continental rise by deep geostrophic contour currents. Science, 152:502-508.

Hein, J. R., O'Neil, J. R., and Jones, M. G., 1979. Origin of authigenic carbonates in sediments from the deep Bering Sea. Sedimentology, 26:681-705.

Iijima, A., 1978, Geological occurrences of zeolite in marine environments. In Sand, L. S., and Mumpton, F. A. (Eds.), Natural Zeolites, Occurrence, Properties, Use: Oxford (Pergamon Press), pp. 175-198.

Irwin, H., Curtis, C. D., and Coleman, M., 1977. Isotopic evidence for source of diagenetic carbonates formed during burial of organic-rich sediments. Nature, 269:209-213.

Krishnaswami, S., 1976. Authigenic transition elements in Pacific pelagic clays. Geochim. Cosmochim. Acta, 40:425-434.

Lancelot, Y., and Ewing, J. I., 1972. Correlation of natural gas zonation and carbonate diagenesis in Tertiary sediments from the Northwest Atlantic. In Hollister, C. D., and Ewing, J. I., et al., Init. Repts. DSDP, 11: Washington (U.S. Govt. Printing Office), 791-799. 
Matsumoto, R., 1978. Occurrence and origin of authigenic $\mathrm{Ca} \cdot \mathrm{Mg} \cdot \mathrm{Fe}$ carbonates and carbonate rocks in the Paleogene coalfield regions in Japan. J. Fac. Sci. Imp. Univ. Tokyo, Sect. 2, 19:335-367.

Matsumoto, R., and lijima, A., 1980. Carbonate diagenesis in cores from Sites 438 and 439 off Northeast Honshu, Northwest Pacific, Leg 57, Deep Sea Drilling Project. In Scientific Party, Init. Repts. DSDP, 56, 57, Pt. 2: Washington (U.S. Govt. Printing Office), 1117-1131.

1981. Origin and diagenetic evolution of $\mathrm{Ca} \cdot \mathrm{Mg} \cdot \mathrm{Fe}$ carbonates in some coalfields of Japan. Sedimentology, 28:239-259. , in press. Chemical sedimentology of some Permo-Jurassic and Tertiary bedded cherts in central Honshu, Japan. In Iijima, A., Siever, R., and Hein, J. (Eds.), Siliceous Deposits in the Pacific Region, Developments in Sedimentology: Amsterdam (Elsevier).

Matsumoto, R., Utada, M., and Kagami, H., 1978. Sedimentary petrology of DSDP cores from Sites 362 and 363, the Walvis Ridge, and Site 364, the Angola Basin, drilled on Leg 40. In Bolli, H. M., Ryan, W. B. F., et al., Init. Repts. DSDP, 40: Washington (U.S. Govt. Printing Office), 469-485.

Milliman, J. D., and Müller, J., 1973. Precipitation and lithification of magnesian calcite in the deep-sea sediments of the eastern Mediterranean Sea. Sedimentology, 20:29-45.
Sayles, F. L., Manheim, F. T., and Waterman, L. S., 1972. Interstitia water studies on small core samples, Leg 11. In Hollister, C. D., Ewing, J. I., et al., Init. Repts. DSDP, 11: Washington (U.S. Govt. Printing Office), 997-1008.

Scholl, P. A., 1971. Diagenesis of deep-water carbonate turbidites, Upper Cretaceous Monte Antola flysch, Northern Apennines, Italy. J. Sediment. Petrol., 41:233-250.

Smythe, J. A., and Dunham, K. C., 1947. Ankerite and Chalybites from the northern Pennine orefield and the north-east coalfield. Min. Mag., 28:53-75.

Taylor, R. K., and Spears, D. A., 1967. An unusual carbonate band in the East Pennine coalfield (England). Sedimentology, 9:55-73.

Thompson, G., and Bowen, V. T., 1969. Analyses of coccolith ooze from the deep tropical Atlantic. J. Mar. Res., 27:32-38.

Zemmels, I., Cook, H. E., and Hathaway, J. C., 1972. X-ray mineralogy studies-Leg 11. In Hollister, C. D., Ewing, J. I., et al., Init. Repts. DSDP, 11: Washington (U.S. Govt. Printing Office), 729-789.

Date of Initial Receipt: May 19, 1982 